\title{
Checklist of Stomatopoda (Malacostraca: Hoplocarida) deposited in the MOUFPE collection, with a new record from Brazil
}

\author{
Débora Lucatelli, Luis E. A. Bezerra, Paulo J. P. dos Santos and Petrônio A. Coelho ${ }^{\dagger}$
}

(DL) (PJPS) (PAC) Universidade Federal de Pernambuco, Programa de Pós-Graduação em Oceanografia, Departamento de Oceanografia. Av. Arquitetura s/n, Cidade Universitária. 50740550, Recife, Pernambuco, Brazil. ${ }^{\dagger}$ in memoriam. E-mail: (DL) dla.lucatelli@gmail.com

(LEAB) Universidade Federal Rural do Semi-Árido (UFERSA), Departamento de Ciências Animais. Av. Francisco Mota, 572. 99625-900 Mossoró, Rio Grande do Norte, Brazil, and Programa de Pós-Graduação em Ciências Marinhas Tropicais, Instituto de Ciências do Mar (LABOMAR), Universidade Federal do Ceará. E-mail: luiseab@gmail.com

\section{Abstract}

A total of 39 species of Stomatopoda were previously reported from marine and estuarine habitats of Brazilian waters. The present checklist is based on material deposited in the crustacean collection of Museu de Oceanografia Petrônio Alves Coelho, Universidade Federal de Pernambuco, city of Recife, Brazil (MOUFPE). The collection, deriving primarily from the northern and northeastern coast of Brazil, includes 667 samples and 1.301 specimens, distributed in four superfamilies, seven families, 14 genera and 33 species, including one holotype and two paratype. Apparent distribution gaps for five-species were filled. Two Brazilian endemic species had their southern geographical ranges increased (Neogonodactylus moraisi (Fausto Filho \& Lemos de Castro, 1973) and Nannosquilla dacostai Manning, 1970) and one was recorded for the first time from northeast Brazilian waters [Alima neptuni (Linnaeus, 1768)]. There are now 42 species of Stomatopoda recorded from the Brazilian coast.

Key words: Biodiversity, Gonodactyloidea, Lysiosquilloidea, northern and northeastern Brazil, Squilloidea.

\section{Introduction}

The crustaceans of the order Stomatopoda, commonly known as mantis shrimps, are among the most aggressive crustacean predators and those with the most complex behavior (Ahyong and Harling, 2000). They are cryptic, benthic animals that are most abundant in marine depths less than about $200 \mathrm{~m}$, although some occur in shallow brackish habitats, and others to $1500 \mathrm{~m}$ depth in soft or hard substrates (Ahyong et al., 2008). Ahyong et al. (2011) considered for the extant Stomatopoda to include one suborder, Unipeltata Latreille, 1825, with seven superfamilies: Bathysquilloidea Manning, 1967; Gonodactyloidea Giesbrecht, 1910; Erythrosquilloidea Manning and Bruce, 1984; Lysiosquilloidea Giesbrecht, 1910; Squilloidea Latreille, 1802; Eurysquilloidea Manning, 1977; and Parasquilloidea Manning, 1995. These taxa comprise 115 genera and 460 species.

Among thesevensuperfamilies recognized by Ahyong et al. (2011), six are recorded in Brazil: Bathysquilloidea, with Bathysquillidae 
Manning, 1967; Gonodactyloidea, including the families Hemisquillidae Manning, 1980, Gonodactylidae Giesbrecht, 1910, Odontodactylidae Manning, 1980 and Pseudosquillidae Manning, 1977; Lysiosquilloidea, including Lysiosquillidae Giesbrecht, 1910 and Nannosquillidae Manning, 1980; Squilloidea with Squillidae Latreille, 1802; Eurysquilloidea comprising Eurysquillidae Manning, 1977 and Parasquilloidea with Parasquillidae Manning, 1995 (Gomes Corrêa, 1998; Schram, 2010). In the last decades few studies were published in Brazil, dealing with distribution, diversity and systematic issues (Barreto et al., 2003; Coelho and Santos, 2003; Silva et al., 2003; Rodrigues and Young, 2005; Almeida et al., 2007a; 2007b; 2008; Bento et al., 2008; Albuquerque and Coelho, 2009; Bento and Melo, 2010; Lucatelli et al., 2012). Nevertheless, these are check-lists for restricted areas or papers dealing with new species. Thus, additional study would be useful in order to achieve a more thorough knowledge of the group in Brazil. The scientific collections are important sources to improve these issues and answer some taxonomic, phylogenetic and biogeographic questions.

There are three most important carcinological collections in Brazil that represent the Brazilian stomatopod fauna: Museu de Zoologia of Universidade de São Paulo (MZUSP), Museu Nacional of Rio de Janeiro (MNRJ) and Museu de Oceanografia Petrônio Alves Coelho of Universidade Federal de Pernambuco (MOUFPE). This paper reviews the identification of species deposited in the Museu de Oceanografia Petrônio Alves Coelho, Universidade Federal de Pernambuco, city of Recife, Brazil (MOUFPE, previously DOUFPE) and provides new records of Stomatopoda from Brazilian waters.

\section{Material and Methods}

The present checklist includes species found from the Cape Orange, northern Amapá State $\left(04^{\circ} 17^{\prime} \mathrm{N} / 51^{\circ} 32^{\prime} \mathrm{W}\right)$ to the Abrolhos
Archipelago, Southern Bahia State (18¹9'S / $\left.39^{\circ} 40^{\prime} \mathrm{W}\right)$. Oceanic areas include the Fernando de Noronha Archipelago (03⒌'S / 35⒉ $5^{\prime} \mathrm{W}$ ), located $345 \mathrm{~km}$ east of the coast of Rio Grande do Norte State, and São Pedro and São Paulo Archipelago $\left(0^{\circ} 55^{\prime} \mathrm{N} / 29^{\circ} 20^{\prime} \mathrm{W}\right)$, located about $500 \mathrm{~km}$ northeast of Fernando de Noronha Archipelago (Fausto Filho, 1974; Holthuis et al., 1980; Leão and Dominguez, 2000). It also includes species from the Rocas Atoll (03ㄴ $45^{\prime}-$ $\left.03^{\circ} 56^{\prime} \mathrm{S} / 33^{\circ} 37^{\prime}-33^{\circ} 56^{\prime} \mathrm{W}\right), 260 \mathrm{~km}$ east of Natal city, Rio Grande do Norte (Kikuchi, 2000), the only atoll in the Southwestern Atlantic, as well as from the seamounts of the North chain (i.e., = seamounts of Ceará) $\left(01^{\circ} 00^{\prime}-04^{\circ} 00^{\prime} S\right.$ / 37 $\left.37^{\circ} 00^{\prime}-39^{\circ} 00^{\prime} \mathrm{W}\right)$ and of Fernando de Noronha (i.e., = seamounts of Fernando de Noronha) $\left(03^{\circ} 00^{\prime}-4^{\circ} 30^{\prime} \mathrm{S}\right.$ / $\left.32^{\circ} 00^{\prime}-37^{\circ} 00^{\prime} \mathrm{W}\right)$, where the homonym archipelago and the Rocas Atoll are located (Coelho Filho, 2006) (Fig. 1).

Material deposited in the crustacean collection of the MOUFPE was mainly identified by Petronio A. Coelho, and reviewed by Débora Lucatelli. This collection was started in the late 1950s and presently is the third largest carcinological collection in Brazil, with more than 14,000 samples (Coelho et al., 2008). The collection contains Stomatopoda mostly obtained during surveys along the continental shelf and littoral areas. Several oceanographic expeditions, however, also collected samples on the upper continental slope. The MOUFPE collection includes almost all Brazilian species and all species known from north and northeast regions.

The following information is included for each species: the type material (when available), the list of material examined (number of specimens, station, date, geographical coordinates, depth, voucher/ catalogue number), distribution, habitat and remarks (when necessary). The synonymies are limited to original papers (containing species description) and the first author list of references.

Classification of the species in families and higher taxa was based on Ahyong et al. (2011). The families within each superfamily 


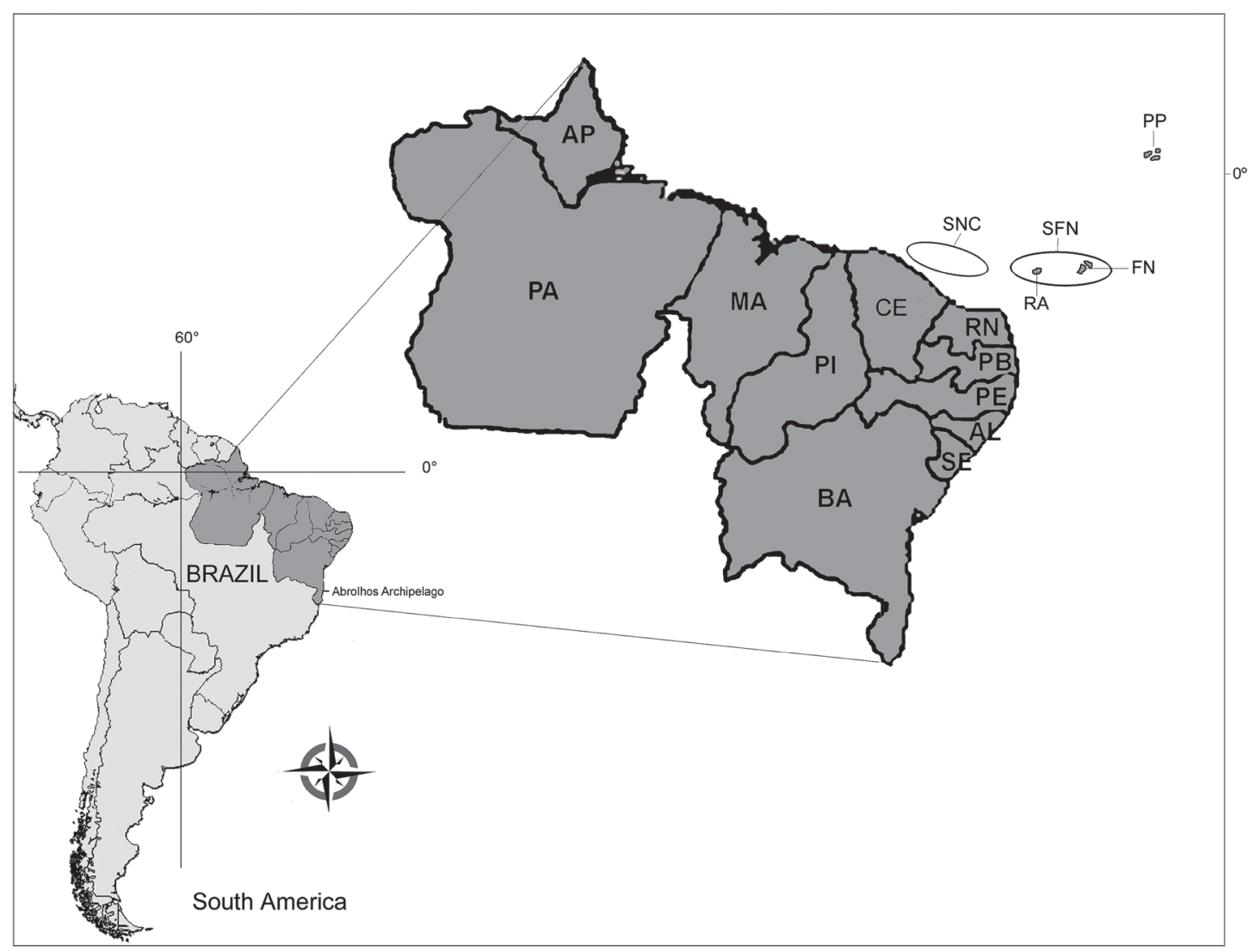

Figure 1. The study area. Abbreviations: Amapá (AP), Pará (PA), Maranhão (MA), Piauí (PI), Ceará (CE), Rio Grande do Norte (RN), Paraíba (PB), Pernambuco (PE), Alagoas (AL), Sergipe (SE), Bahia (BA); Oceanic Islands and Seamounts: seamounts of north chain (SNC), seamounts of Fernando de Noronha (SFN), Fernando de Noronha Archipelago (FN), São Pedro and Sáo Paulo Archipelago (PP), Rocas Atoll (RA) (modified from Coelho et al. 2008).

and the species within each family follow Ahyong et al. (2011). Morphological terminology used for species descriptions follows Ahyong (2001).

Abbreviations: north region states: Amapá (AP) and Pará (PA); northeast region states: Maranhão (MA), Piauí (PI), Ceará (CE), Rio Grande do Norte (RN), Paraíba (PB), Pernambuco (PE), Alagoas (AL), Sergipe (SE) and Bahia (BA); oceanic islands and seamounts: seamounts of north chain (SNC), seamounts of Fernando de Noronha (SFN), Fernando de Noronha Archipelago (FN), São Pedro and São Paulo Archipelago (PP) and Rocas Atoll (RA). Oceanographic expeditions: Akaroa (AKA), Almirante Saldanha (SALD), Camamu Bay (BCAM), Petrobrás Bacia Potiguar first expeditition (BPot I), Petrobrás Bacia Potiguar second expeditition (BPot II), Petrobrás Bacia Potiguar third expeditition (BPot III), Petrobrás Bacia Potiguar fourth expeditition (BPot IV), Petrobrás Bacia
Potiguar Regional Grid (MR), Petrobrás Bacia Potiguar - Programa de Avaliação de Impacto (BPot-PAI), Petroleum drillings on Potiguar Basin (RRN, RNS, CES), Canopus (CAN), Agência Estadual de Planejamento e Pesquisa de Pernambuco Projetc (CONDEPE/Suape); Geomar (GM), Itamaracá (ITA), Manati petroleum drillings - Camamu basin - Bahia (MANATI), Pesquisador IV program Maranhão (PESQ. IV), Programa de Avaliação dos Recursos Vivos da Zona Econômica Exclusiva (REVIZEE ), REVIZEE program score Northeast first expedition (NE I), REVIZEE program score Northeast second expedition (NE II), REVIZEE program score Northeast third expedition (NE III), REVIZEE program score Northeast fourth expedition (NE IV), Recife (REC) and Pavasas expeditition (PAVASAS). Museum collections: Australian Museum (AM); Laboratório de Ciências do Mar, Universidade Federal do Ceará, Fortaleza, Brazil (LABOMAR); 
Muséum National d'Histoire Naturelle, Paris, France $(\mathrm{MNHN})$; National Museum of Natural History, Smithsonian Institution, Washington DC, United States of America (USNM).

\section{Results}

The Stomatopoda collection includes 667 samples and 1,301 specimens, distributed in four superfamilies (Gonodactyloidea, Lysiosquilloidea, Squilloidea and Eurysquilloidea), seven families (Gonodactylidae, Odontodactylidae, Pseudosquillidae, Lysiosquillidae, Nannosquillidae, Squillidae and Eurysquillidae), 14 genera and 33 species, including one holotype and two paratypes.

\section{Systematics}

Subclass Hoplocarida Calman, 1904

Order Stomatopoda Latreille, 1817

Suborder Unipeltata Latreille, 1825

Superfamily Gonodactyloidea Giesbrecht, 1910

Family Gonodactylidae Giesbrecht, 1910

Genus Neogonodactylus Manning, 1995

Neogonodactylus austrinus (Manning, 1969)

Gonodactylus austrinus Manning, 1969: 338, fig. 91 - Coelho and Koening, 1972: 251.

Neogonodactylus austrinus - Manning, 1995: 80; Gomes-Corrêa, 1998: 293; Schram, 2010: 59.

Material examined: Holotype (Abrolhos, Brazil; 2-5 m) (MNHN-St 198) 28 November 1961; 2 paratypes (0350'30"S / 3350'40"W; $28 \mathrm{~m}$ ) (MNHN-St 188) 17 November 1961; 2 paratypes (Calypso, South America; 7-8 m) (MNHN-St 187) 17 November 1961; 1 paratype (0349'25"S / 32 24'25”W; $24 \mathrm{~m}$ ) (MNHN-St 190), 18 November 1961; 4 specimens (FN; 03051'S / 32。25'W) (MOUFPE 978) 31 July 1963; 1 specimen, (REC 3; 0809'S / 34049’W; 22 m) (MOUFPE 979) 23 July 1967; 3 specimens (PE 27A; 07050’08”S / 34042'00”W; 30 m) (MOUFPE 981) 06 February 1969; 3 specimens (ITA 81; 07038'S / 34047'W; 2 m) (MOUFPE 982); 2 specimens (CAN 14; 01³0'S / 3848'W) (MOUFPE 983) 12 July 1965; 2 specimens (REC 19; 086'S / 34052'W; 23 m) (MOUFPE 984); 1 specimen (ITA 87; 07037'S / 34047’W; 0.7 m) (MOUFPE 985) 06 February 1969; 1 specimen (Porto de Galinhas beach-PE; 08³0'S / 3500'W; reef), (MOUFPE 986) 01 August 1975; 1 specimen (MA 24; 01053'S / 4248'W; 43 m) (MOUFPE 987); 1 specimen (Ponta das Pedras Pretas beach-PE; 0817'S / 34056'W; subtidal zone) (MOUFPE 988) 14 September 1962; 4 specimens, (AKA 42; 09³2'S / 35²0'W; $31 \mathrm{~m}$ ) (MOUFPE 989); 5 specimens (ITA 46; 07043'S / 34048'W; 3.7 m) (MOUFPE 990); 1 specimen (SALD 1647A; 07030'00"S / 34³4'07"W; 37 m) (MOUFPE 991) 06 October 1967; 6 specimens (ITA 73; 07041'S / 34048'W; 4 m) (MOUFPE 992); 2 specimens (CAN 109; 04045'S / 36025'W; $45 \mathrm{~m}$ ) (MOUFPE 993) 15 January 1966; 3 specimens (REVIZEE-NE III 40; 4005'38'S / 37025'34”W; 50 m) (MOUFPE 12346) 15 May 1998; 1 specimen (REVIZEE-NE III 77); 1'37'30"S / 38०06'29"W; 43 m) (MOUFPE 12555) 07 June 1998; 1 specimen (REVIZEENE III 93; 03०45'04”S / 32¹9'58”W; 70 m) (MOUFPE 12559) 02 June 1998; 1 specimen (BPot IV 38-dredge; 04047.50'S 3650.22’W; 15 m) (MOUFPE 14055) 29 May 2004.

Distribution: Western Atlantic Caribbean Coast of Honduras, Nicaragua, Panama and Brazil (Fernando de Noronha and Abrolhos Archipelago, São Pedro and São Paulo Archipelago, Rocas Atoll and Maranhão to Espírito Santo) (Gomes-Corrêa, 1998).

Habitat. It was found among calcareous algae, rocks and in coral reefs, at depths from shallow subtidal to $70 \mathrm{~m}$. These records are in agreement with Manning (1969), who reported this species to $73 \mathrm{~m}$ depth.

Remarks: The specimens agree with holotype description. The number of antennal articles was analysed, but it is highly variable and can not be used as a diagnostic character, the same was observed by Manning (1969). The number of movable spines varied from 11 
to 12 on the specimens analysed, a similar range was found in other species of Neogonodactylus.

\section{Neogonodactylus lacunatus (Manning, 1966)}

Gonodactylus lacunatus Manning, 1966: 374, fig. 6c-Manning, 1969: 311, fig. 86; Coelho and Koening, 1972: 251; Gomes-Corrêa, 1998: 119.

Neogonodactylus lacunatus - Manning, 1995: 80; Rodrigues and Young, 2005: 243; Almeida et al., 2008: 25; Schram, 2010: 63.

Material examined: 2 paratypes (USNM 113251) (Abrolhos Archipelago, Brazil, $\left.18^{\circ} 00^{\prime} \mathrm{S} / 38^{\circ} 18^{\prime} \mathrm{W} ; 48 \mathrm{~m}\right) 28$ November 1961; 3 paratypes (Abrolhos Archipelago, Bahia, Brazil, 18²18'S / 38 53'W; 38 m) (USNM 113252) 29 November 1961; 2 specimens (Gaibú-PE; 08¹8'S / 34º56'W; subtidal zone) (MOUFPE 1044) 24 July 1993; 2 specimens (ITA 17; 07051'S / 34049'W; 4 m) (MOUFPE 1069); 4 specimens (REC 3; 0809'S / 34049'W; 22 m) (MOUFPE 1070); 11 specimens (REC 119; 08¹3'S / 34049'W; 26 m) (MOUFPE 1071); 8 specimens (ITA 43; 07044'S / 34²9'W; 2 m) (MOUFPE 1073); 1 specimen (CONDEPE/Suape 2-PE) (08²1'S I 34057'W) (MOUFPE 1074) 02 March 1978; 1 specimen (Santa Cruz Cabrália-BA; 16¹7'S / 39॰02'W) (MOUFPE 1075) 21 October 1962; 1 specimen (Tambaú beachPB; 07007'S / 34º52'W) (MOUFPE 1076) 27 September 1971; 1 specimen (Piedade-PE; 08¹0'S / 34056'W) (MOUFPE 1077) 02 September 1960; 3 specimens (SALD 1732A; 02¹3'S / 41'051'W; 69 m) (MOUFPE 1079); 1 specimen (SALD 1719A; 02²1'S / 40²9'W; $37 \mathrm{~m}$ ) (MOUFPE 1080); 1 specimen (PB/PE 28B; 07041'S / 34035'W) (MOUFPE 1081); 2 specimens (PE 34); 07040'S / 34043'W; 19 m) (MOUFPE 1082) 04 March 1969; 6 specimens (Fortaleza-CE; 0343'S / 38³0'W; subtidal zone) (MOUFPE 1083) 04 July 1965; 1 specimen (REC 2; 0809'S / 34050'W; 18 $\mathrm{m})$ (MOUFPE 1084); 2 specimens damaged (AKA 3; 0856'S / 34º57'W; 36 m) (MOUFPE 1085); 4 specimens (Tambaú-PB; 07007’S / 34052'W; subtidal zone) (MOUFPE 1086) 13 April 1968; 5 specimens (AKA 48; 09037'S /
352 $5^{\prime} \mathrm{W} ; 30$ m) (MOUFPE 1087); 1 specimen (Porto de Galinhas-PE; 08³0'S / 3500'W; subtidal zone) (MOUFPE 1088) 06 April 1973; 13 specimens (ITA 74; 07041'S / 34047’W; 5 m) (MOUFPE 1089); 1 specimen (AKA 85; 10¹2'S / 36 00'W; $21 \mathrm{~m}$ ) (MOUFPE 1090); 1 specimen (CAN 21; 0321'S / 38³8'W; 25 m) (MOUFPE 1091) 16 July 1965; 7 specimens (PE 15B; 08²0'S / 34046'W; 35 m) (MOUFPE 1093); 1 specimen (REC 94; 080.'S / 34046'W; 26 m) (MOUFPE 1094); 4 specimens damaged (PE 34A; 07040'S / 34043'W; 19 m) (MOUFPE 1095) 04 March 1969; 7 specimens (REC 136; 0802'S / 34043'W; $31 \mathrm{~m}$ ) (MOUFPE 1096); 6 specimens (PE 29B; 07044'S / 34046'W; 14 m) (MOUFPE 1097) 25 February 1969; 1 specimen (SALD 1721A; 02²0'S / 4047'W; 35 m) (MOUFPE 1098); 2 specimens (SALD 1656; 0541'S / 3505'W; $23 \mathrm{~m}$ ) (MOUFPE 1099); 4 specimens (PE 35; 07039'S / 34º37'W; 32 m) (MOUFPE 1100) 07 March 1969; 3 specimens (AKA 100; 10²8'55"S / 36¹5'30"W; 25 m) (MOUFPE 1102); 3 specimens (PE 31; 07044'S / 34³7’W; 33 m) (MOUFPE 1103) 07 March 1969; 1 specimen, (AKA several trawls) (MOUFPE 1104); 2 specimens (ITA 82; 07³8'S / 34047'W; 2.5 m) (MOUFPE 1105); 8 specimens (REC 140; 08 $19^{\prime}$ 'S / 34053'W; $19 \mathrm{~m}$ ) (MOUFPE 1106); 2 specimens (CAN 12; 0313'S / 38³1'W; 45 m) (MOUFPE 1107) 01 July 1965; 4 specimens (CAN 109; 04045'S / 36²5'W; 45 m) (MOUFPE 1108) 15 January 1966; 2 specimens (Pina Riverdredging-PE; 0804'S / 34\%54'W; subtidal zone) (MOUFPE 1109) 28 March 1964; 7 specimens (AKA 37; 09²7'S / 3517’W; 32 m) (MOUFPE 1110); 2 specimens damaged (AKA 43; 09032'S / 35¹5’W; 36 m) (MOUFPE 1111); 1 specimen (REC 82; 0810'S / 3451'W; 15 m) (MOUFPE 1112); 3 specimens (PESQ. IV 22; 0209'S / 42०52'W) (MOUFPE 1113); 1 specimen (AKA 41; 09032'S / 35²5'W; 26 m) (MOUFPE 1114); 1 specimen (AKA 40; 0932'S / 35०30'W; 16 m) (MOUFPE 1115); 4 specimens (PE 29A; 07044'S / 34046'W; 14 m) (MOUFPE 1116) 25 February 1969; 7 
specimens (SALD 1655; 0604'S / 34059'W; $25 \mathrm{~m}$ ) (MOUFPE 1117); 1 specimen (AKA 71; 09॰58'S / 35॰47'W; 34 m) (MOUFPE 1118); 1 specimen (AKA 53; 09041'S / 528'W; 36 m) (MOUFPE 1119); 3 specimens (REC 144; 08²0'S / 3448'W; 33 m) (MOUFPE 1120); 1 specimen (ITA 14; 07051'S / 34049'W; 2 m) (MOUFPE 1121); 13 specimens (PE 29D; 07044'S / 34046'W; $14 \mathrm{~m}$ ) (MOUFPE 1122) 25 February 1969; 2 specimens (PE 29; 07045'S / 34046'W; 14 m) (MOUFPE 1123) 25 February 1969; 2 specimens (AKA 93; 10²1'S / 3605'W; 27 m) (MOUFPE 1124); 2 specimens (ITA 10; 07050'S / 34049'W; 4 m) (MOUFPE 1126); 4 specimens (REC 47/13; 0809'S / 34051'W; $15 \mathrm{~m}$ ) (MOUFPE 1127) 13 June 1966; 5 specimens (CONDEPE/Suape 2; 08²1'S / 34057'W) (MOUFPE 1128) 28 February 1978; 6 specimens (REC 127; 0805'S / 34045'W; 23 m) (MOUFPE 1129); 8 specimes (AKA 49; 09॰37'S / 35॰30'W; 23 m) (MOUFPE 1130); 8 specimens (ITA 91; 07035'S / 34048'W; 1.5 m) (MOUFPE 1131); 4 specimens (ITA 16A; 07051'S / 34049'W; 4 m) (MOUFPE 1133); 1 specimen (REC 109; 07058'S / 34042'W; 29 m) (MOUFPE 1134) 24 February 1967; 2 specimens (ITA 44; 07044'S / 34049'W; 1.5 m) (MOUFPE 1135); 1 specimen (MA 24; 0153'S / 42048'W; 43 m) (MOUFPE 1136); 13 specimens (SALD 1647A; 07030'S / 34o34.7’W; 37 m) (MOUFPE 1138) 06 October 1967; 25 specimens (SALD 1730; 02³7'S / 41이W; $21 \mathrm{~m}$ ) (MOUFPE 1139); 2 specimens (Ponta Verde-AL; 0940'S / 35॰43'W; subtidal zone) (MOUFPE 1140), 11 August 1972; 4 specimens (SALD 1751; 00³7'S / 44040'W; $44 \mathrm{~m}$ ) (MOUFPE 1141); 14 specimens (Olinda-PE; 0801'S / 34\%51'W, subtidal zone) (MOUFPE 1142) 01 June 1964; 1 specimen (Fortaleza-CE; 0343'S / 38³0'W; subtidal zone) (MOUFPE 1143) 02 July 1965; 2 specimens (SALD 1722; 02013'30"S / 40॰43'30”W; 53 m) (MOUFPE 1144) 29 October 1967; 1 specimen (AKA 117; 10॰42'45”S / 36³1'45”W; 27 m) (MOUFPE 1145) 10 August 1965; 3 speciemens (CAN 95; 0545'S / 34058'W; 45 m) (MOUFPE
1146) 13 January 1966; 4 specimens (AKA 32; 09०24'15"S / 35०14'10"W; 27 m) (MOUFPE 1147) 08 September 1965; 1 specimen (Porto de Galinhas-PE; 08³0'S / 3500’W; subtidal zone) (MOUFPE 1148) 09 August 1975; 6 specimens (PB/PE 13; 07004'S / 34045'W) (MOUFPE 1149); 1 specimens (JaguaribeITA-PE; 07043'S / 34050'W; subtidal zone) (MOUFPE 1150) 12 December 1970; 1 specimen damaged (Tamandaré-PE; 0844'S / 3506'W; subtidal zone) (MOUFPE 1151) 01 July 1972; 5 specimens (PE 35; 07039'S / 34\%37'W; $32 \mathrm{~m}$ ) (MOUFPE 1152) 07 March 1969; 3 specimens (SALD 1662A; 03053'50"S / 33०46'40”W; 25 m) (MOUFPE 1276) 09 October 1967; 3 specimens (REC 63; 0809'S / 34048'W; $23 \mathrm{~m}$ ) (MOUFPE 5394); 1 specimen (PE 17B; 08¹0'S / 3451'W; $15 \mathrm{~m}$ ) (MOUFPE 8438) 22 January 1969; 1 specimen (REC 95; 08¹0'S / 34²5'W; $31 \mathrm{~m}$ ) (MOUFPE 8440); 1 specimen (Santo Aleixo Island-PE 01; 8³6'42.37”S / 3501'27.64”W; subtidal zone) (MOUFPE 13509) 06 February 2007; 2 specimens (PAVASAS I 03-dredge; 02॰46'S / 39॰30'W; 25 m) (MOUFPE 13579) 19 July 1987; 19 specimens (Bpot IV 38-dredge; 04047.50'S / 36050.22'W; 15 m) (MOUFPE 14051) 29 May 2004; 3 specimens (Bpot IV 35-dredge; 04047.48'S / 3644.81'W; 19.7 m) (MOUFPE 14059) 28 May 2004; 1 specimen (Bpot IV 14-dredge; 4047.41'S / 36²6.89'W; $27 \mathrm{~m}$ ) (MOUFPE 14081) 27 May 2004; 1 specimen (Bpot IV A8N; 4044'30.5”S / 36033'44.6”W; $30.36 \mathrm{~m}$ ) (MOUFPE 14082) 21 June 2004; 1 specimen juvenile (Bpot IV 17-dredge; 04057.67'S / 36²5.93'W; $13.7 \mathrm{~m}$ ) (MOUFPE 14083) 27 May 2004; 6 specimens (BPot IV A2N; 4058'06.8”S / 36²'37.3”W; $11 \mathrm{~m}$ ) (MOUFPE 14084) 20 June 2004; 2 specimens (Bpot IV 30-dredge; 04047.46'S / 36³9.38'W; $22.5 \mathrm{~m}$ ) (MOUFPE 14085) 28 May 2004; 1 specimen (Bpot IV 38-VanVeen A; 04047.50'S / 36050.22'W; $15 \mathrm{~m}$ ) (MOUFPE 14086) 20 May 2004; 1 specimen-juvenile (Bpot IV 21-dredge; 04058.30'S / 36³3.94’W; $11 \mathrm{~m}$ ) (MOUFPE 14088) 28 May 2004; 7 specimens (Bpot IV A10; 4048’38.40”S / 3642’15”W; 
17 m) (MOUFPE 14089) 22 May 2004; 1 specimen (Bpot IV 15-dredge; 04052.87'S / 360 28.55'W; 14.6 m) (MOUFPE 14097) 27 May 2004; 2 specimens (BPot IV A1N; 4058'08.3"S / 36º'17'25.8”'W; $10.2 \mathrm{~m}$ ) (MOUFPE 14098) 20 June 2004; 1 specimen (Bpot IV A11; 4047'41.6”S / 3647'46.04”W; 13.6 m) (MOUFPE 14099) 22 May 2004; 1 specimen juvenile (Bpot IV 12-dredge; 0452.84'S / 36²3.17'W; 18.5 m) (MOUFPE 14100) 26 May 2004; 7 specimens (BPot IV A5N; 4'37'33.83"S / 36०50'11.4”W; 84.9 m) (MOUFPE 14102) 21 June 2004; 1 specimen (Bpot IV 38-Van Veen C; 04047.50'S 3650.22'W; $15 \mathrm{~m}$ ) (MOUFPE 14103) 29 May 2004; 2 specimens (BPot IV A2N; 4058'06.8”S / 36²'37.3”W; $11 \mathrm{~m}$ ) (MOUFPE 14104) 20 June 2004; 1 specimen (Bpot IV A7; 4044'54”S / 3652'25”W; 12.2 m) (MOUFPE 14105) 20 June 2004; 2 specimens (Bpot IV E24; 0502.27's / 36²3.13'W; 6 m) (MOUFPE 14106) 22 May 2004; 1 specimen (Bpot IV A3A; 4049'58'S / 36¹2'12”; $41.7 \mathrm{~m}$ ) (MOUFPE 14111) 23 May 2004; 3 specimens (Bpot III 42-dredge; 04042'09”'S / 36055.63'W; 20.2 m) (MOUFPE 14125) 24 November 2003; 2 specimens (Bpot III 35-dredge; 040 47.48'S / 3644.81'W; $15 \mathrm{~m}$ ) (MOUFPE 14126) 24 November 2003; 4 specimens (Bpot III 14-dredge; 04047.41'S / 36²6.89'W; $27 \mathrm{~m}$ ) (MOUFPE 14127) 23 November 2003; 1 specimen (Bpot III 17-dredge; 04057.67'S / 36²5.93'W; 11.2) (MOUFPE 14128) 22 November 2003; 7 specimens (Bpot III 30-dredge; 04047.46'S / 36039.38'W; $18 \mathrm{~m}$ ) (MOUFPE 14129) 23 November 2003; 1 specimen (Bpot III MF 29; 500'19.1"S / 36²6'55”W; $5.1 \mathrm{~m}$ ) (MOUFPE 14160) 23 November 2003; 3 specimens (Bpot III MF 35; 500'19.1"S / 36²6'55”W; $5.1 \mathrm{~m}$ ) (MOUFPE 14161) 22 November 2003; 2 specimens (Bpot III 09; 0504.75'S / 36¹8.93'W; $1.6 \mathrm{~m}$ ) (MOUFPE 14162) 16 November 2003; 2 specimens (Bpot III MF 37; 501'54.5”S / 36³1'08.1”W; $5.3 \mathrm{~m}$ ) (MOUFPE 14163) 21 November 2003; 1 specimen (Bpot III MF 31; 500'56.5” S / 36²4'18.4”W; 5.7 m) (MOUFPE 14164) 22
November 2003; 3 specimens (Bpot III MF 39; 5001'55.4”S / 36³1'52.1”W; 4.9 m) (MOUFPE 14165) 21 November 2003; 1 specimen (Bpot III MF 32; 500'08.4”S / 36 24'16.3"W; $5.9 \mathrm{~m}$ ) (MOUFPE 14167) 22 November 2003; 2 specimens (Bpot II 21B; 04'58.30'S / 36³3.94'W; 9.6 m) (MOUFPE 14186); 1 specimen (Bpot II 35B-dredge; 04047.48'S / 36²4.81'W; 15.8 m) (MOUFPE 14187); 1 specimen (Bpot II MF 15-trawl; 405'23.3”S / 36040'11.5”W; $21.4 \mathrm{~m}$ ) (MOUFPE 14188) 28 May 2003; 7 specimens (Bpot II 14C-dredge; 04047.41'S / 36²6.89'W; $27 \mathrm{~m}$ ) (MOUFPE 14190); 4 specimens (Bpot II 07-trawl; 04058.23'S / 36º 17.73'W; $10.5 \mathrm{~m}$ ) (MOUFPE 14191) 27 May 2003; 1 specimen (Bpot II 17-trawl; / 04057.67'S 36²5.93'W; 9.9 m) (MOUFPE 14192) 29 May 2003; 1 specimen (Bpot II 30Van Veen A; 04047.46'S / 36'39.38'W; 18.8 m) (MOUFPE 14193) 20 May 2003; 1 specimen (BPot II 03-dredge; 04051.43'S / 36¹2.36'W; $29.1 \mathrm{~m}$ ) (MOUFPE 14194) 22 May 2003; 1 specimen (Bpot II MF 14-trawl; 4057'50.6”S / 36³6'18.3”W; 7.5 m) (MOUFPE 14197) 28 May 2003; 1 specimen (Bpot II 15C-dredge; 04052.87'S / 36²8.55'W; $11.8 \mathrm{~m}$ ) (MOUFPE 14198) 22 May 2003; 2 specimen (Bpot II 15-dredge; 0452.87'S / 36²8.55'W; 11.8 m) (MOUFPE 14199) 22 May 2003; 4 specimens (BPot II 39-dredge; 04042.08'S / 3650.23'W; $15.1 \mathrm{~m}$ ) (MOUFPE 14216) 19 May 2003; 1 specimen (BPot II MF 10-trawl; 4048'38.40"S / 36042'15”W; $17 \mathrm{~m}$ ) (MOUFPE 14217) 28 May 2003; 2 specimens (Bpot II 22-dredge; 04º52.88'S / 36³3.98'W; 13.3 m) (MOUFPE 14218) 23 May 2003; 2 specimens (BPot II 18A-dredge; 04058.29'S / 36²8.53'W; 8.6 m) (MOUFPE 14222); 2 specimens (Bpot II 38-dredge; 04047.50'S / 3650.22'W; $11.1 \mathrm{~m}$ ) (MOUFPE 14223) 19 May 2003; 3 specimens (Bpot II 14-dredge; 04047.41'S / 36²6.89'W; 27 m) (MOUFPE 14225) 23 May 2003; 1 specimen (Bpot II 13C-dredge; 04047.41'S / 36²3.17'W; $47.1 \mathrm{~m}$ ) (MOUFPE 14226); 2 specimens (BPot II 24-dredge; 04041.09'S / 36³4.03'W; 67.1 m) (MOUFPE 14228) 24 
May 2003; 6 specimens (BPot II 40-dredge; 04036.66'S / 3650.25'W; $79.1 \mathrm{~m}$ ) (MOUFPE 14230) 19 May 2003; 1 specimen (Bpot II 11A; 04058.25'S / 36²7.14'W; $10.2 \mathrm{~m}$ ) (MOUFPE 14232); 1 specimen (BPot II 11C-dredge; 04058.25'S / 36º27.14'W; 10.2 m) (MOUFPE 14233); 1 specimen (BPot II 13-dredge; 0447.41'S / 36²3.17’W; $47.1 \mathrm{~m}$ ) (MOUFPE 14237) 23 May 2003; 1 specimen (BPot II 21-dredge; 0458.30'S / 36033.94’W; $9.6 \mathrm{~m}$ ) (MOUFPE 14239) 21 May 2003; 1 specimen (BPot II 35-dredge; 04047.48'S / 36044.81'W; $15.8 \mathrm{~m}$ ) (MOUFPE 14241) 20 May 2003; 1 specimen (BPot II 14B-dredge; 04047.41'S / 36²6.89'W; $27 \mathrm{~m}$ ) (MOUFPE 14242); 6 specimens (BPot II 17B-trawl; 04057.67'S / 36º25.93'W; 9.9 m) (MOUFPE 14243); 1 specimen (BPot I 29A-dredge; 04053.66'S / 36040.44'W; 12 m) (MOUFPE 14244); 1 specimen (Bpot I 29C-dredge; 04053.66'S / 36040.44'W; 12 m) (MOUFPE 14245); 5 specimens (BPot I 35B-dredge; 04047.48'S / 3644.81'W; 15.8 m) (MOUFPE 14246); 10 specimens (BPot I 37A-dredge; 04057.83'S / 36044.24'W; 8 m) (MOUFPE 14247) August 2002; 1 specimen (Bpot I 21B-dredge with algae; 04058.30'S / 36033.94'W; $9.6 \mathrm{~m}$ ) (MOUFPE 14248); 2 specimens (BPot I 29-dredge; 04053.66'S / 3640.44'W; $12 \mathrm{~m}$ ) (MOUFPE 14249); 6 specimens (BPot I 18C-dredge; 04058.29'S / 36²8.53'W; $8.6 \mathrm{~m}$ ) (MOUFPE 14251) August 2002; 1 specimen (BPot I 35C-dredge; 04047.48'S / 3644.81'W; 15.8 m) (MOUFPE 14252); 1 specimen (BPot I 22B-dredge; 0452.88'S / 36³3.98'W; 13.3 m) (MOUFPE 14254); 1 specimen (BPot I 13B; 04047.41'S / 36²3.17'W; $47.1 \mathrm{~m}$ ) (MOUFPE 14255); 1 specimen (BPot I 13A-dredge; 04047.41'S / 36²3.17'W; $47.1 \mathrm{~m}$ ) (MOUFPE 14256); 1 specimen (BPot I 21C-dredge; 04058.30'S / 36033.94'W; 9.6 m) (MOUFPE 14.257); 1 specimen (BPot I 30C-dredge; 04047.46'S / 36³9.38'W; $18.8 \mathrm{~m}$ ) (MOUFPE 14260) August 2002; 1 specimen (BPot I 14C-dredge; 4047.41'S / 36²6.89'W; 27 m) (MOUFPE 14262); 1 specimen (BPot I 30; 04047.46'S / 36039.38'W; $18.8 \mathrm{~m}$ ) (MOUFPE 14263)
August 2002; 1 specimen (BPot I 22A-dredge; 0452.88'S / 36³3.98'W; 13.3 m) (MOUFPE 14264); 1 specimen (BPot I 30B-dredge with algae; 04047.46'S / 36³9.38'W; $18.8 \mathrm{~m}$ ) (MOUFPE 14267) August 2002; 1 specimen (BPot I 37C-dredge; 04057.83'S / 3644.24'W; $8 \mathrm{~m}$ ) (MOUFPE 14269) July-August 2002; 2 specimens (BPot MF 35; 04047.48'S / 36044.81'W; $15.8 \mathrm{~m}$ ) (MOUFPE 14878) 22 November 2003; 1 specimen damaged (BPot MF 31; 500'56.5”S / 36²4'18.4”W; 5.7 m) (MOUFPE 14879) 22 November 2003; 1 specimen juvenile (BPot MF 24; 502'00.3”S / 36¹4'09.4”W; 8.4 m) (MOUFPE 14880) 23 November 2003; 15 specimens (BPot III 38-dredge; 04047.50’S / 36050.22'W; $11.1 \mathrm{~m}$ ) (MOUFPE 14881) 24 November 2003; 2 specimens (Carneiros beach 03 with rocks and Porifera; 0842'45”S / 3504'33”W, coral reefs) (MOUFPE 14882) 15 June 2007; 2 specimens (PE 03; 08047’05”S / 34055'W; 36 m) (MOUFPE 14883) 24 August 2004.

Distribution: Western Atlantic Mexico (Yucatan), Nicaragua, Virgin Islands, Colombia (Santa Marta area: Bahía Concha; Punta Ancón), Brazil (Fernando de Noronha and Abrolhos Archipelago, Maranhão to Rio de Janeiro) (Gomes-Corrêa, 1998).

Habitat. It was found in coral reef locations, calcareous algae and seagrass beds from shallow subtidal to $85 \mathrm{~m}$ (Manning, 1969). Our records are in agreement with Manning (1969), and the lower depth limit is slightly increased from 80 to $85 \mathrm{~m}$.

Remarks: The uropodal endopod outline of type series and additional material studied herein are almost straight instead of sinuous as Manning (1969) reported. Furthermore, most of specimens analysed in this study do not bear a movable apex on submedian tooth of telson, but in some the apex was present, including the female paratype (TL $16-28 \mathrm{~mm}$ ), changing the diagnosis given by Manning (1969).

Neogonodactylus minutus (Manning, 1969)

Gonodactylus minutus Manning, 1969: 304307, fig. 84 - Coelho and Koening, 1972: 251; Gomes-Corrêa, 1998: 294. 
Neogonodactylus minutus - Manning, 1995: 80; Schram, 2010: 64.

Material examined: Holotype (Recife, Brazil; $52 \mathrm{~m}$ ) (USNM 113247); 1 paratype (0348'35"S / 32²4'50"W; 52 m) (MNHNSt 182) 18 November 1961; 1 specimen (AKA 04; 08056'15”S / 34052'40”W; 44 m) (MOUFPE 1265) September 1965; 1 specimen (AKA 16; 09¹1'S / 3502'W; 41 m) (MOUFPE 1266); 2 specimens (AKA 05; 09॰01'00"S / 34051'10"W; $46 \mathrm{~m}$ ) (MOUFPE 1267) 10 September 1965; 4 specimens (REC 155; 08¹3'08”S / 34053'06”W; $15.5 \mathrm{~m}$ ) (MOUFPE 1268) 07 March1967; 1 specimen (SALD 1701A; 01057'00"S / 37046'00"W; $57 \mathrm{~m}$ ) (MOUFPE 1269) 21 October 1967; 1 specimen (AKA 44; 09032'05"S / 35०10’35”W; $40 \mathrm{~m})$ (MOUFPE 1270) 08 September 1965; 1 specimen (REC 149; 07056'07"S / 34037'02"W; 38 m) (MOUFPE 1271) May 1967; 2 specimens (CAN 39; 04033'S / 3700'W) (MOUFPE 1272); 1 specimen (SALD 1722; 02¹3'30"S / 40॰43'30”W; 53 m) (MOUFPE 1273) 30 October 1967; 1 specimen (AKA 35; 0927’S / 3507'W; 32 m) (MOUFPE 6794); 1 specimen (SALD 1647A; 07030'S / 34034.7'W; $37 \mathrm{~m}$ ) (MOUFPE 6795) 06 October 1967; 3 specimens (CAN 81; 08¹0'S / 34³5'W) (MOUFPE 6796); 1 specimen (CAN 19; 01056'S 37051'W) (MOUFPE 6797); 2 specimens damaged (PE 15B; 08¹3'04”S / 34º51'07”W; 21.5 m) (MOUFPE 6798) 10 May 1966; 3 specimens (REVIZEE-NE II 112/111; 3०50'17"S / 33041'13"W; 28 m) (MOUFPE 12332) 03 March 1997; 1 specimen (BPot MF 33; 0500'04.7”S / 36²5’07.4”W; $6 \mathrm{~m}$ ) (MOUFPE 14896) 22 November 2003.

Distribution: Western Atlantic Brazil (Fernando de Noronha and Trindade Archipelago, Ceará to Rio de Janeiro) (Manning, 1969).

Habitat: Shallow water to $95 \mathrm{~m}$ (GomesCorrêa 1998), among calcareous algae and on sandy bottoms.

Remarks: The specimens analysed in this study agree very well with the description given by Manning (1969).
Neogonodactylus moraisi (Fausto Filho and Lemos de Castro, 1973)

Gonodactylus moraisi Fausto Filho and Lemos de Castro, 1973: 61, fig. 1A-D. - Fausto Filho and Sampaio Neto, 1976: 66; Schotte and Manning, 1993: 567; Gomes-Corrêa, 1998: 294.

Neogonodactylus moraisi - Manning, 1995: 80; Schram, 2010: 64.

Material examined: 1 specimen (REVIZEE-NE III 40; 405'38”S / 37०25’34”W; $50 \mathrm{~m}$ ) (MOUFPE 12348) 15 May 1998; 1 specimen (BPot IV 38-dredge; 04047.50'S / 3650.22'W; $15 \mathrm{~m}$ ) (MOUFPE 14054) 29 May 2004.

Distribution: Western Atlantic - Brazil (Amapá, Ceará) (Fausto Filho and Lemos de Castro, 1973). The species is herein reported from Rio Grande do Norte State, extending its southernmost distribution limit within Brazil.

Habitat: Sandy bottoms, between 15-76 $\mathrm{m}$ depth. The previous depth range (50-76 m; Fausto Filho and Lemos de Castro, 1973), is extended to $15 \mathrm{~m}$.

Remarks: The holotype is now lost, but the paratype is in MNRJ (MNRJ 5547) and should be checked to confirm some characters that were not figured in the original account. The specimens agree well with description provided by Fausto Filho and Lemos de Castro (1973).

\section{Neogonodactylus oerstedii (Hansen, 1895)}

Gonodactylus oerstedii Hansen, 1895: 65 Rathbun, 1935: 113; Holthuis, 1941: 38; Manning, 1961: 43, pl. 11, figs. 5-6; 1963: 422, tabs. 3-4; 1969: 325, fig. 89; 1977: 282; Schotte and Manning, 1993: 571; GomesCorrêa, 1998: 294.

Neogonodactylus oerstedii - Manning, 1995: 80; Hendrickx and Salgado-Barragán, 2002: 393; Schram, 2010: 64.

Material examined: 3 specimens (PE 35A; 07039'S / 34º37'W; 32 m) (MOUFPE 994) 07 March 1969; 3 specimens (SALD 1944; 20³7’S / 34042'W; 80-131 m) 
(MOUFPE 1233); 4 specimens (SALD 1718; 0207'S / 40³6'W; $71 \mathrm{~m}$ ) (MOUFPE 1234) 28 October 1967; 1 specimen (SALD 1667B; 03050.8'S / 32०27.5’W; 55 m) (MOUFPE 1235) 10 October $1967 ; 1$ specimen (SALD 1946; 20³2'S / 35०46'W; 70 m) (MOUFPE 1236) 08 September 1968; 1 specimen (AKA 32; 09²4'S / 3514'W; 27 m) (MOUFPE 1237) 08 September 1965; 1 specimen (ITA 92; 07035'S / 34098'W; 1.5 m) (MOUFPE 1238) 20 February 1969; 1 specimen (PiedadePE; 0809'51"S / 3454'41"W; subtidal zone) (MOUFPE 1239) 12 November 1960; 2 specimens (CAN 19; 01056'S / 37051'W; $48 \mathrm{~m}$ ) (MOUFPE 1242); 1 specimen (CAN 41; 0427'S / 37004'W; 58 m) (MOUFPE 1243) 23 August 1965; 1 specimen (CAN 48; 040.''s / 37º33'W) (MOUFPE 1244) August 1965; 2 specimens (SALD 1767A; 01057.5’ N / 47013.5'W; $93 \mathrm{~m}$ ) (MOUFPE 1245) 13 November 1967; 1 specimen (SALD 1662B; 03050'03"S / 33०45'60”W; $47 \mathrm{~m}$ ) (MOUFPE 1246) 09 October 1967; 1 specimen (Pina-PE; 08004'S / 34054'W; subtidal zone) (MOUFPE 1247) 14 September 1962; 3 specimens (Paripe-Bahia; 1259'S / 38०31W; subtidal zone) (MOUFPE 1248); 1 specimen (REVIZEE-NE III 93; 3045'04"S / 32¹9'58"W; 70 m) (MOUFPE 12597) 02 June 1998; 2 specimens (BPot IV 38-dredge; 04047.50'S / 36०50.22'W; 15 m) (MOUFPE 14052) 29 May 2004; 1 specimen (BPot IV 35-dredge; 04047.48'S / 3644.81'W; 19.7 m) (MOUFPE 14056) 28 May 2004; 1 specimen (Bpot IV E22; 0502.39'S / 36²3.12'W; $7.4 \mathrm{~m}$ ) (MOUFPE 14061) 23 May 2004; 1 specimen (BPot IV 14-dredge; 4047.41'S / 36²6.89’W; $27 \mathrm{~m}$ ) (MOUFPE 14115) 27 May 2004; 1 specimen (BPot III 38-dredge; 04047.50'S / 3650.22'W; $11.1 \mathrm{~m}$ ) (MOUFPE 14143) 24 November 2003; 1 specimen (BPot III MF 24; 0502'00.3”S / 36¹4’09.4”W; 8.4 m) (MOUFPE 14172) 23 November 2003; 2 specimens (Bpot II 705) (MOUFPE 14221); 1 specimen (Bpot III MF 31; 500'56.5”S / 36²4'18.4”W; $5.7 \mathrm{~m}$ ) (MOUFPE 14871) 22 November 2003; 1 specimen (BPot MF 39; 501'55.4”S / 36³1'52.1”W; 4.9 m) (MOUFPE 14872) 21 November 2003; 1 specimen (Bioincrustação-P2 10B R1 C2; 12057'S / 38³0'W; biofouling) (MOUFPE 14873) December 2008; 1 specimen (Bioincrustação-P4 5S R1 C2; 12057’S / 38³0'W; biofouling) (MOUFPE 14874) December 2008; 3 specimens (BPot-PAI 121 RNS 31 Extra; 04²6.8399'S / 36²5.4041'W; $46.6 \mathrm{~m}$ ) (MOUFPE 14875) 13 July 2009; 1 specimen (BPot-PAI 121 RNS 22 Extra; 04046'58”S / 36²5'21"W; 43 m) (MOUFPE 14876) 15 July 2009; 1 specimen (BPotRegional MR 42 R1 Extra; 04045'54"S / 36²5'49”W; $52 \mathrm{~m}$ ) (MOUFPE 14977) 25 October 2009; 1 specimen (BPot-Regional MR 25 R3; 04052'17”S / 36045'58”W; 12 m) (MOUFPE 14978).

Distribution: Western Atlantic Bermuda, Bahamas, Florida, Mexico, Aruba, Bonaire, Curaçao, Venezuela, Trinidad, Brazil (Rocas Atoll and Fernando de Noronha Archipelago, Amapá to São Paulo) (Holthuis, 1941; Schotte and Manning, 1993; GomesCorrêa, 1998).

Habitat: Coral reef and seagrass, at depths from less than $5 \mathrm{~m}$ to $131 \mathrm{~m}$. The lower distribution limit provided by Gomes-Corrêa (1998: $120 \mathrm{~m}$ ) is increased to $131 \mathrm{~m}$.

\section{Neogonodactylus spinulosus (Schmitt, 1924)}

Gonodactylus oerstedii var. spinulosus Schmitt, 1924: 96, pl. 5, fig. 5.

Gonodactylus spinulosus - Manning, 1961: 41, pl. 11, figs. 3-4; 1969: 299, fig. 83; Schotte and Manning, 1993: 570; Gomes-Corrêa, 1998: 294.

Neogonodactylus spinulosus - Manning, 1995: 80; Hendrickx and Salgado-Barragán, 2002: 393; Rodrigues and Young, 2005: 241; Schram, 2010: 66.

Material examined: Holotype (off Castle, east side Barbados) (USNM 68945); 3 specimens (SALD 1662A; 0353'S / 334' 'W; 25 m) (MOUFPE 1252) 09 October 1967; 5 specimens (SALD 1662B; 0350'03"S / 33०45'60"W; $47 \mathrm{~m}$ ) (MOUFPE 1253) 09 October 1967; 3 specimens (SALD 1944; 20³7'00”S / 34042'00”W; 80-131 m) (MOUFPE 1254) 07 September 1968; 6 
specimens (CAN 90; 06035'S / 34044'W; $54 \mathrm{~m}$ ) (MOUFPE 1256) 21 December 1965; 1 specimen (SALD 1668A; 03053'S / 32०37.2W; $60 \mathrm{~m}$ ) (MOUFPE 1257) 10 October 1967; 3 specimens (SALD 1663; 03०49,6'S / 33०49.2W; $53 \mathrm{~m}$ ) (MOUFPE 1258) 09 October 1967; 1 specimen (CAN 78; 08³4'S / 34044'W) (MOUFPE 1260); 1 specimen (SALD 1682; 03²8'S / 3506. ' 'W; $61 \mathrm{~m}$ ) (MOUFPE 1261) 17 October 1967; 2 specimens (CAN 18; 01056'S / 37051'W) (MOUFPE 1262); 1 specimen (SALD 1667B; 03050.8'S / 32027.5'W; 55 m) (MOUFPE 1263) 10 October 1967; 4 specimens (SALD 1667A; 03050.7’S / 32²8.1'W; 65 m) (MOUFPE 1264) 10 October 1967; 3 specimens (REVIZEE-NE III 81A; 3707'16"S / 1043'48”W; 55 m) (MOUFPE 12344) 06 June 1998; 1 specimen (REVIZEENE III 88; 03049'37”S / 34042'47”W; 55 m) (MOUFPE 12551) 04 June 1998; 1 specimen (REVIZEE-NE III 77A; 1³7'30”S / 38०06'29"W; $43 \mathrm{~m}$ ) (MOUFPE 12554) 07 June 1998; 1 specimen (REVIZEE-NE III 85; 3०28'01"S / 35०03'40”W; $62 \mathrm{~m}$ ) (MOUFPE 12556) 04 June 1998; 6 specimens (REVIZEENE III 93; 3045'04” / 32०19'58”W; 70 m) (MOUFPE 12560) 02 June 1998; 1 specimen (REVIZEE-NE III 96; 4015'32"S / 33॰14'35”W; 49 m) (MOUFPE 12562) 01 June 1998; 3 specimens (REVIZEE-NE III 28; 7o27'11"S / 34³1'30"W; 43 m) (MOUFPE 12590) 10 May 1998; 1 specimen (REVIZEENE III 90A; 3०48'29”S / 33०40'58”W; 70 m) (MOUFPE 12901) 03 June 1998.

Distribution: Western Atlantic - Mexico, Bermuda and southern Florida, Tobago, Bahamas, Belize, Haiti, Puerto Rico, Virgin Islands, St. Martin, Barbuda, St. Eustatius, Antigua, Guadeloupe, Dominica, Aruba, Panama, Barbados, Trinidad, Bonaire, Klein Bonaire, Curaçao, Brazil (Rocas Atoll and Fernando de Noronha Archipelago, Ceará to Rio de Janeiro) (Rodrigues and Young, 2005).

Habitat: In coral rubble and calcareous algae bottom, known from 0 to $130 \mathrm{~m}$ depth (Gomes-Corrêa, 1998).

Remarks: Some specimens have spaced dark patches of chromatophores on the sixth thoracic somite and first abdominal somite in agreement with Manning (1969).

Neogonodactylus torus (Manning, 1969)

Gonodactylus torus Manning, 1969: 90, fig. 90 - Coelho and Koening, 1972: 251; GomesCorrêa, 1998: 294.

Neogonodactylus torus - Manning, 1995: 80; Manning and Heard, 1997: 303; Hendrickx and Salgado-Barragán, 2002: 393; Almeida et al., 2007b: 7; 2008: 25; Schram, 2010: 67.

Material examined: Holotype (Palm Beach, Florida, USA) (USNM 119289); 2 specimens (AKA 91; 10¹9'S / 36¹5'W; 15 m) (MOUFPE 1241); 1 specimen (AKA 46; 0937'S / 3515'W; 36 m) (MOUFPE 1249); 1 specimen (PB 22; 07021'S / 34041'W; 28 m) (MOUFPE 1250); 2 specimens (SALD 1783A; 0322'N / 485' $\mathrm{W} ; 105 \mathrm{~m}$ ) (MOUFPE 1251) 16 November 1967; 1 specimen (Carneiros beach-PE; 08²'S / 35005’ W, coral reef) (MOUFPE 8715) July 1997; 1 specimen (REVIZEE-NE I 213; 4039'43"S / 36043'23"W; 52 m) (MOUFPE 12340); 1 specimen (REVIZEE-NE II 109/108; 3048'S / 3312'W) (MOUFPE 12586) 03 March 1997; 1 specimen (REVIZEE-NE III 166; 8०48'S / 35²'W) (MOUFPE 12899) 15 July 1998; 1 specimen (Santo Aleixo Island-PE 01; 8³6'42.37”S / 3501'27.64”W; subtidal zone in rocks) (MOUFPE 13433) June 2006; 1 specimen (Santo Aleixo Island-PE 01; 8³6'42.37”S / 3501'27.64”W; subtidal zone in rocks) (MOUFPE 13510) February 2007; 1 specimen (BPot IV 38-dredge; 04047.50'S / 36०50.22'W; $15 \mathrm{~m}$ ) (MOUFPE 14057) 29 May 2004; 2 specimens (BPot IV 05-dredge; 0447.40'S / 36º17.77'W; 85 m) (MOUFPE 14065) 30 May 2004; 2 specimens (BPot IV 24-dredge; 04041.09'S / 36034.03'W; $72 \mathrm{~m}$ ) (MOUFPE 14080) 05 June 2004; 2 specimens (BPot IV A23; 04047.42'S / 36033.97’W; $21.8 \mathrm{~m}$ ) (MOUFPE 14087) 05 June 2004; 2 specimens (BPot IV 24-dredge; 0441.09'S / 36³4.03'W; 72 m) (MOUFPE 14101) 24 May 2004; 1 specimen (BPot IV 14-dredge; 4047.41'S / 36²6.89'W; 27 m) (MOUFPE 14110) 27 May 2004; 
1 specimen (BPot IV A2N; 4058'06.8"S

/ 3612’37.3”W; $11 \mathrm{~m}$ ) (MOUFPE 14112) 20 June 2004; 1 specimen (BPot IV A5N; 4037'33.83"S / 36050'11.4”W; 84.9 m) (MOUFPE14114) 21 June 2004; 1 specimen (BPot IV A1N; 4058'08.3"S / 36²17'25.8”W; $10.2 \mathrm{~m}$ ) (MOUFPE 14117) 20 June 2004; 2 specimens (BPot III 13-dredge; 04047.41'S / 36²3.17’ W; $45.7 \mathrm{~m}$ ) (MOUFPE 14130) 22 November 2003; 2 specimens (BPot III 06-dredge; 04052.84'S / 36º 17.83'W; 34.5 m) (MOUFPE 14159) 21 November 2003; 1 specimen (Bpot III MF 29; 501'57.9"S / 36¹9'32.7”W; $5.7 \mathrm{~m}$ ) (MOUFPE 14166) 23 November 2003; 1 specimen (BPot III 39-dredge; 04042.08S / 36050.23W; 17.60 m) (MOUFPE 14168) 24 November 2003; 1 specimen (BPot III MF 39; 501'55.4”S / 36³1'52.1"W; 4.9 m) (MOUFPE 14169) 21 November 2003; 1 specimen (BPot III 05; 04047.40’S / 36²17.77’W; 93 m) (MOUFPE 14170) 14 November 2003; 2 specimens (BPot III 21-dredge; 0458.30'S / 36³3.94’W; 10.7 m) (MOUFPE 14171) 22 November 2003; 4 specimens (BPot II 40-dredge; 04036.66'S / 3650.25'W; $79.1 \mathrm{~m}$ ) (MOUFPE 14215) 19 May 2003; 3 specimens (BPot II 13-dredge; 04047.41'S / 36²3.17’W; 47.1 m) (MOUFPE 14220) 23 May 2003; 2 specimens (BPot II 24-dredge; 04041.09'S / 36³4.03’W; $67.1 \mathrm{~m}$ ) (MOUFPE 14229) 24 May 2003; 1 specimen (BPot II 13-dredge; 04047.41'S / 36²3.17’ W; $47.1 \mathrm{~m}$ ) (MOUFPE 14238) 23 May 2003; 5 specimens (BPot I 13A-dredge; 04047.41'S / 36²3.17'W; $47.1 \mathrm{~m}$ ) (MOUFPE 14259); 1 specimen (REVIZEE IV-NE 149; 6¹5'36"S / 34053' 17”W; $30 \mathrm{~m}$ ) (MOUFPE 14888) 24 November 2000; 2 specimens (BPotPAI 121 RNS 34 Extra; 04046'56"S / 36²5'32”W; $44.3 \mathrm{~m}$ ) (MOUFPE 14889) 14 July 2009; 1 specimen (Santo Aleixo IslandPE 01; 08³6'42.37”S / 3501'27.64”W; subtidal zone) (MOUFPE 14890) 19 April 2007; 1 specimen (BPot-PAI 121 RNS 42 Extra; 04046'58”S / 36²5'08”W; 45.7 m) (MOUFPE 14891) 15 July 2009; 1 specimen (BPot-PAI 121 RNS 33 Extra; 04047'07”S / 36²5'24”W; $40 \mathrm{~m}$ ) (MOUFPE 14892) 14 July 2009; 1 specimen (BPot-PAI 121 RNS
43 Extra; 04047'15”S / 36²5'24”W; 38.2 m) (MOUFPE 14893) 14 July 2009; 1 specimen (BPot-PAI 121 RNS 21 R3 (0-10); 04046'55"S / 36025'24”W; 45.7 m) (MOUFPE 14894) 13 July 2009.

Distribution: Western Atlantic - North Carolina, Georgia, Florida, Cuba, Mexico, Panama, Barbados and Brazil (Amapá to Bahia) (Gomes-Corrêa, 1998).

Habitat. Rocky reef bottom and inside sponge cavity (Gomes-Corrêa, 1998), at depths from 10 to $364 \mathrm{~m}$ (Manning, 1969).

Remarks: The specimens analysed herein showed some morphological variations, as on rostral plate (extending or not until the base of ocular peduncle), carina swollen and telson's proportion. Manning (1969) described males with more swollen carinae than females, but the males analysed in the present contribution present swollen or slender carinae. The apical spinule was found in some males, although this was not mentioned by Manning (1969) or Ahyong and Norrington (1997).

Neogonodactylus wennerae Manning and Heard, 1997

Gonodactylus oerstedii- Lunz, 1935:152, fig. 1. [not G. oerstedii Hansen, 1895].

Gonodactylus bredini - Manning, 1969: 315, figs. 88a-c [part, see Manning and Heard, 1996]; Camp, 1973: 53, figs. 21-26, tabs. 14-17, plate 1 [part, see Manning and Heard, 1997]; Fausto Filho and Sampaio Neto, 1976: 66; Manning, 1977: 280; Morgan and Goy, 1987: 595-618; Schotte and Manning, 1993: 567; Gomes-Corrêa, 1998: 293. [not $G$. bredini Manning, 1969].

Neogonodactylus bredini - Manning, 1995: 80; Almeida et al., 2008: 24.

Neogonodactylus wennerae Manning and Heard, 1997: 306, figs. 2, 3 [detailed loc. list]; - Hendrickx and Salgado-Barragán, 2002: 393; Schram, 2010: 67.

Material examined: Holotype (United States; off South Carolina; 32 49'18”N / 78³9'24”W; $44 \mathrm{~m}$ ) (USNM 232666) 03 November 1981; 1 specimen (PiedadePE; 08¹0'S / 34\%56'W, subtidal zone) 
(MOUFPE 1011) 10 September 1991; 1 specimen (REVIZEE-NE III 40; 4005'38”S / 37०25'34”W; 50 m) (MOUFPE 12347) 15 May 1998; 1 specimen (REVIZEENE III 53; 0114'24”S / 4114'24”W) (MOUFPE 12553) 16 May 1998; 4 specimens (REVIZEE-NE III 36; 5029'53"S / 35०04'41"W; $24 \mathrm{~m}$ ) (MOUFPE 12588) 14 May 1998; 1 specimen (REVIZEE-NE III 28; 7²7'11”S / 34³1'30”W; 43 m) (MOUFPE 12592) 10 May 1998; 1 specimen (REVIZEENE III 42; 0329'46”S / 38०08'53”W; 19 m) (MOUFPE 12598) 16 May 1998; 5 specimens (REVIZEE-NE III 166; 8०48'S / 35¹2'W) (MOUFPE 12923) 15 July 1998; 1 specimen (Santo Aleixo Island-PE 01 836'42.37'S / 3501'27.64"W; subtidal zone in rocks) (MOUFPE 13508) 06 February 2007; 3 specimens (BPot IV 38-dredge; 04\%47.50'S / 3650.22'W; 15 m) (MOUFPE 14053) 19 May 2004; 1 specimen (Bpot IV 35-dredge; 04047.48'S / 36॰44.81'W; 19.7 m) (MOUFPE 14058) 28 May 2004; 1 specimen (Bpot IV E22; 0502.39'S / 36²3.12'W; $7.4 \mathrm{~m}$ ) (MOUFPE 14068) 23 May 2004; 3 specimens (BPot IV A2N; 4058'06.8”S / 36¹2'37.3”W; $11 \mathrm{~m}$ ) (MOUFPE 14079) 20 June 2004; 1 specimen (Bpot IV A7; 4044'54"S / 36०52'25”W; 12.2 m) (MOUFPE 14107) 20 May 2004; 1 specimen (BPot IV A5N; 4037'33.83"S / 3650'11.4”W; 84.9 m) (MOUFPE 14108) 21 June 2004; 1 specimen (Bpot IV A7N; 4044'54"S / 36052'25"W; $12.2 \mathrm{~m}$ ) (MOUFPE 14109) 20 June 2004; 1 specimen (Bpot III 19-dredge; 0503.51'S / 36²8.55'W; $3.9 \mathrm{~m}$ ) (MOUFPE 14140) 19 November 2003; 1 specimen (Bpot III 42-dredge; 04042'09”S / 36055.63'W; 20.2 m) (MOUFPE 14141) 24 November 2003; 1 specimen (Bpot III 35-dredge; 040 47.48'S / 36044.81'W; $15 \mathrm{~m}$ ) (MOUFPE 14142) 24 November 2003; 1 specimen (Bpot III 14 dredge; 4047.41'S / 36²6.89'W; $28 \mathrm{~m}$ ) (MOUFPE 14144) 23 November 2003; 1 specimen (Bpot III 21-dredge; 04058.30'S / 36³3.94'W; $10.7 \mathrm{~m}$ ) (MOUFPE 14157) 22 November 2003; 1 specimen (Bpot III MF 34; 501'03.9” / 36²5’05.6”; $7.1 \mathrm{~m}$ ) (MOUFPE 14158) 23 November 2003; 3 specimens (Bpot
III 17-trawl; 04057.67'S / 36º 25.93'W; 11.2 m) (MOUFPE 14173) 22 November 2003; 1 specimen (Bpot II MF 14-trawl; 4057'50.6"S / 36036'18.3”W; $7.5 \mathrm{~m}$ ) (MOUFPE 14196) 28 May 2003; 1 specimen (BPot II 15-dredge; 04 52.87'S / 36²8.55'W; $11.8 \mathrm{~m}$ ) (MOUFPE 14200) 22 May 2003; 1 specimen (BPot II 30-dredge; 04047.46'S / 36º39.38'W; $18.8 \mathrm{~m}$ ) (MOUFPE 14219) 20 May 2003; 1 specimen (Bpot II 38-dredge; 04047.50'S / 3650.22’W; $11.1 \mathrm{~m}$ ) (MOUFPE 14224) 19 May 2003; 2 specimens (BPot II 09-dredge; 0504.75'S / 36¹8.93'W; $2.8 \mathrm{~m}$ ) (MOUFPE 14227) 15 May 2003; 9 specimens (BPot II 40-dredge; 04036.66'S / 3650.25'W; 79.1 m) (MOUFPE 14231) 19 May 2003; 1 specimen (BPot II MF 11-trawl; 04047'41.6”S / 36047'46.04”W; 13.6 m) (MOUFPE 14234) 28 May 2003; 1 specimen (Bpot II 13-dredge; 04047.41'S / 36²3.17'W; $47.1 \mathrm{~m}$ ) (MOUFPE 14236) 23 May 2003; 1 specimen (BPot II 21-dredge; 0458.30'S / 36³3.94'W; 9.6 m) (MOUFPE 14240) 21 May 2003; 1 specimen (BPot I 37B-dredge; 04057.83'S / 3644.24'W; $8 \mathrm{~m}$ ) (MOUFPE 14250) August 2002; 4 specimens (BPot I 35 B-dredge; 04047.48'S / 3644.81'W; $15.8 \mathrm{~m}$ ) (MOUFPE 14253) August 2002; 4 specimens (BPot I 22A-dredge; 04052.88'S / 36033.98'W; $13.3 \mathrm{~m}$ ) (MOUFPE 14261) August 2002; 1 specimen (BPot I 37C-dredge; 04'57.83'S / 36044.24'W; 8 m) (MOUFPE 14265) August 2002; 2 specimens (BPot I 30A dredge; 04047.46'S / 36039.38'W; $18.8 \mathrm{~m}$ ) (MOUFPE 14268) August 2002; 1 specimen (Paracuru beach-CE; 03०24'03.71"S / 39०00'51.32"W; subtidal zone) (MOUFPE 14877) 11 July 2002; 3 specimens (Bpot III 38-dredge; 04047.50’s / 36050.22’W; $11.1 \mathrm{~m}$ ) (MOUFPE 14884) 24 November 2003; 1 specimen (Bpot III 03; 04051.43'S / 36¹2.36'W; $29.1 \mathrm{~m}$ ) (MOUFPE 14885) 13 November 2003; 1 specimen (BPot MF 37; 04057.83'S / 36044.24'W; 8 m) (MOUFPE 14886) 21 November 2003; 1 specimen (Bpot-PAI 121 RNS 42 extra; 04046'58"S / 36²5'08”W; $45.6 \mathrm{~m}$ ) (MOUFPE 14887) 15 July 2009; 1 specimen (Bpot-Regional MR $32 \mathrm{R} 1 ; 04^{\circ} 49^{\prime} 20^{\prime \prime S ~ / ~ 36 ² 5 ' 56 ” W ; ~} 22 \mathrm{~m}$ ) (MOUFPE 14976) 27 October 2009. 
Distribution: Western Atlantic Bermuda, North Carolina, South Carolina, Gulf of Mexico, Florida to Curaçao and Brazil (Maranhão to Rio de Janeiro) (Schotte and Manning, 1993; Gomes-Corrêa, 1998).

Habitat: Hard substrates such as coral reefs and rock wall, among seagrass and sponges at depths from 0 to $85 \mathrm{~m}$. The lower depth limit provided by Schotte and Manning (1993), $73 \mathrm{~m}$, is herein extended to $85 \mathrm{~m}$.

Remarks: Some of the specimens identified as $N$. bredini by Manning (1969, fig. 88) are actually $N$. wennerae, given the bathymetry (sublittoral), smaller size and the long rostral spine (Manning and Heard, 1997). The presently analysed specimens agree with these features and should thus be recorded as $N$. wennerae instead of $N$. bredini. Some additional variations were found in the present material, such as the presence or absence of movable apex on intermediate denticle and the rostral plate extending or not until de base of ocular peduncle. These features are not conservative and can lead to doubtful records and the last one is probably a preservation effect as the position of the ocular somite can change depending on the degree of contraction of the arthrodial membrane between the ophthalmic and ocular somites.

\section{Neogonodactylus spp.}

Material examined: 1 specimen juvenile (REVIZEE-NE III 93; 3045'04"S / 32०19'58"W; $70 \mathrm{~m}$ ) (MOUFPE 12584) 02 June 1998; 4 specimens juveniles (BPot IV 35-dredge; 04047.48'S / 3644.81'W; 19.7 m) (MOUFPE 14071) 28 May 2004; 1 specimen juvenile (BPot IV 12-dredge; 04052.84'S / 36²3.17’W; $18.5 \mathrm{~m}$ ) (MOUFPE 14078) 26 May 2004; 3 specimens resected (BPot IV 37-dredge; 04057.83'S / 36044.24'W; $7.4 \mathrm{~m}$ ) (MOUFPE 14118) 30 May 2004; 1 specimen juvenile (BPot IV 38-Van Veen B; 04047.50'S 36/ o50.22'W; 15 m) (MOUFPE 14119) 29 May 2004; 1 specimen juvenile (BPot III 14-dredge; 4047.41'S / 36²6.89'W; $27 \mathrm{~m}$ ) (MOUFPE 14139) 23 November 2003; 1 specimen juvenile (BPot II 14-dredge;
04047.41'S / 36²6.89'W; 27 m) (MOUFPE 14235) 03 May 2003; 1 specimen juvenile (BPot I 30B; 04047.46'S / 36039.38'W; $18.8 \mathrm{~m}$ ) (MOUFPE 14266) August 2002; 1 specimen juvenile (BCAM-40 GAR S6 R2 C2; 1329'S / 38०54”W) (MOUFPE 14949); 1 specimen juvenile (BCAM GAR S3 R3 C2; 1329'18"S / 38०54'05"W) (MOUFPE 14950); 1 specimen juvenile (BPot-PAI 121 RNS 21 Extra; 04046'55”S / 36²5'24”W; 45.7) (MOUFPE 14951) 13 July 2009; 1 specimen juvenile (BPot-PAI 121 RNS 22 R3 (0-10); 04046'58”S / 36²5'21”W; $43.6 \mathrm{~m}$ ) (MOUFPE 14952) 15 July 2009; 1 specimen juvenile (BPot-PAI 121 RNS 41 R1 (0-10); 04046'42”S / 36025'24”W; 46.7 m) (MOUFPE 14953) 13 July 2009; 1 specimen juvenile (BCAM-40 GAR B3 R1 C2; 1329'45"S / 38०54'19"W) (MOUFPE 14954); 1 specimen juvenile (BPot-Regional MR 25 R2; 04052'17”S / 36045'58”W) (MOUFPE 14979); 1 specimen juvenile (BPot-Regional MR 41 CL R2; 04049'40"S / 36¹0'06"W; $58 \mathrm{~m}$ ) (MOUFPE 14980) 27 October 2009; 1 specimen juvenile (BPotRegional MR 43 R3-Extra; 04038'28"S / 36045'37”W; $52 \mathrm{~m}$ ) (MOUFPE 14981) 26 October 2009; 1 specimen juvenile (BPotRegional MR 45 CO R2; 04035'17"S / 3655'03”W; $47 \mathrm{~m}$ ) (MOUFPE 14982) 01 May 2010.

Remarks: These specimens are either juveniles or damaged and identification to species level was not possible.

\section{Family Odontodactylidae Manning, 1980 Genus Odontodactylus Bigelow, 1893 \\ Odontodactylus havanensis (Bigelow, 1893)}

Gonodactylus havanensis Bigelow, 1893: 101. Odontodactylus havanensis - Bigelow, 1894: 497, figs. 1-2, pl. 20; Lunz, 1937: 5, fig. 2; Holthuis, 1941: 37; Ahyong, 2001: 79; Rodrigues and Young, 2005: 234; Schram, 2010: 73.

Odontodactylus brevirostris - Manning, 1967: 22, fig. 8; 1969: 285, figs. 80-81; Coelho and Koening, 1972: 251 (in part); Gomes-Corrêa, 1998: 294 . 
Material examined: Holotype (Off Havana, Cuba) (USNM 17997); 1 specimen (Tamandaré beach-PE; 084's'S / 350.' 'W; subtidal zone, in gravel bottom) (MOUFPE 995) 01 July 1972; 1 specimen (SALD 1944; 20³7'S / 34²42'W; 80-131 m) (MOUFPE 996) 07 September 1968; 2 specimens (AKA 104; 10³3'45”S / 36¹2'W; $27 \mathrm{~m}$ ) (MOUFPE 999) September 1965; 1 specimen (GM 218-3D; 0209'N / 47²5’W; 92 m) (MOUFPE 1001); 3 specimens (BPot IV 03-dredge; 04051.43'S / 36²12.36'W; 33.5 m) (MOUFPE 14120) 26 May 2004; 1 specimen (BPot III 11-dredge; 04058.25'S / 36 27.14'W; $10.2 \mathrm{~m}$ ) (MOUFPE 14145) 21 November 2003; 4 specimens (BPot III MF 24; $05^{\circ} 02^{\prime} 00.3$ ”S / 36²14'09.4”W; 8.4 m) (MOUFPE 14175) 23 November 2003; 1 specimen (Bpot III MF 29; 501'57.9"S / 36¹9'32.7”W; $5.7 \mathrm{~m}$ ) (MOUFPE 14176) 23 November 2003; 3 specimens (BPot III MF 26; 501'56.7”S / 36¹6'13.4”W; 7.3 m) (MOUFPE 14179) 23 November 2003; 3 specimens (BPot III MF 25; 501'57.2”S / 36¹5'07.3”W; $6.6 \mathrm{~m}$ ) (MOUFPE 14180) 23 November 2003; 2 specimens (BPot III MF 39; 501'55.4"S / 36³1'52.1"W; 4.9 m) (MOUFPE 14183) 21 November 2003; 1 specimen (BPot II MF 15-trawl; 0405'23.3”S / 36040'11.5"W; 21.4 m) (MOUFPE 14189) 28 May 2003; 1 specimen (BMA-Manati CO $1 \mathrm{R} 2$; 1320'S / 3852'W; $50 \mathrm{~m}$ ) (MOUFPE 14962) February 2009; 1 specimen (BPotRegional MR 43 R3-Extra; 04038'28”S / 36045'37"W; $52 \mathrm{~m}$ ) (MOUFPE 14963) 01 May 2010; 1 specimen damaged (SALD 1755; 00³7.5'S / 45०38.5'W; $80 \mathrm{~m}$ ) (MOUFPE 997) 07 November 1967; 1 specimen (CANOPUS 08A; 02²0'S / 4120'W) (MOUFPE 998) July 1965; 1 specimen (BPot IV 24-dredge; 04041.09'S / 36³4.03'W; 72 m) (MOUFPE 14066) 24 May 2004; 1 specimen (BPot IV 20-dredge; 0503.73'S / 36º33.91'W; $4.2 \mathrm{~m}$ ) (MOUFPE 14074) 20 May 2004; 2 specimens (BPot III 14-dredge; 4047.41'S / 36²6.89'W; 27 m) (MOUFPE 14146) 23 November 2003; 6 specimens (Bpot III MF 34; 501'03.9”S / 36 25'05.6”'W; $7.1 \mathrm{~m}$ ) (MOUFPE 14174) 22 November 2003; 2 specimens (Bpot III
MF 32; 500'08.4”S / 36²4'16.3”W; 5.9 m) (MOUFPE 14177) 22 November 2003; 2 specimens (BPot III MF 27; 501'55.8”S / 36¹7'24.3”W; $2.7 \mathrm{~m}$ ) (MOUFPE 14178) 23 November 2003; 6 specimens (BPot III MF 28; 0501'56.9”S / 36²18'22.9”W; 6.2 m) (MOUFPE 14181) 23 November 2003; 7 specimens (BPot III MF 33; 0500'04.7”S / 36²5’07.4”W; $6 \mathrm{~m}$ ) (MOUFPE 14182) 22 November 2003; 2 specimens (BPot II MF 12-trawl; 4058'26.8”S / 36027'48.8”; $9.6 \mathrm{~m}$ ) (MOUFPE 14195) 28 May 2003; 1 specimen (BPot II MF 07-trawl; 04044'54"S / 3652'25”W; $12.2 \mathrm{~m}$ ) (MOUFPE 14212) 27 May 2003; 1 specimen (BPot II MF 23-trawl; 502'S / 36² 14 'W; $8 \mathrm{~m}$ ) (MOUFPE 14213) 29 May 2003; 1 specimen (BPot I 24-dredge; 04041.09'S / 36³4.03'W; 67.1 m) (MOUFPE 14281) August 2002; 1 specimen (PE 30 R1; 07045'S / 34043'W; 19 m) (MOUFPE 14869) 26 February 1969; 1 specimen (BPot-PAI 121 RNS 22 extra; 04046'58”S / 36²5'21”W; 44 m) (MOUFPE 14870) 15 July 2009.

Distribution: Western Atlantic - Gulf of Mexico to Brazil (Pará to Rio de Janeiro) (Gomes-Corrêa, 1998).

Habitat: In fine sand and calcareous algae bottoms, from 4 to $309 \mathrm{~m}$ (Manning, 1969).

Remarks: Odontodactylus havanensis was removed from the synonymy of $O$. brevirostris by Ahyong (2001), on the basis of characters such as the uropodal exopod pigmentation and presence or absence of a posterolateral spine on AS3. The Western Atlantic population is recognized as $O$. havanensis (see Ahyong, 2001).

\section{Family Pseudosquillidae Manning, 1977 Genus Pseudosquilla Dana, 1852 \\ Pseudosquilla ciliata (Fabricius, 1787)}

Squilla ciliata Fabricius, 1787: 333; Manning, 1963: 311.

Squilla stylifera Lamarck, 1818: 189.

Squilla quadrispinosa Eydoux and Souleyet, 1842: 362, pl. 15, fig. 1.

Pseudosquilla ciliata var. occidentalis Borradaile, 1900: 398. 
Pseudosquilla ciliata - Kemp, 1913: 96; Lunz, 1937: 6; Chopra, 1939: 160; Holthuis, 1941: 35; Lemos de Castro, 1955: 26, figs. 20-21, pl. 7 and 16; Manning, 1961: 39, pl. 11, figs. 1-2; Manning, 1968: 42; 1969: 264, fig. 74; 1970: 264; Coelho and Koening, 1972: 251; Fausto Filho and Sampaio Neto, 1976: 66; Manning, 1977: 100, figs. 30, 31, 55; Reaka and Manning, 1987: 182, tab. 1; Holmquist, et al. 1989: 255, tab. 2; Moosa, 1991: 169; Richer de Forges and Moosa, 1992: 155, figs. 2, 5, tabs. 1-4; Schotte and Manning, 1993: 571; Manning, 1995: 59; Gomes-Corrêa, 1998: 295; Moosa, 2000: 426; Ahyong, 2001: 112, fig. 55; 2002: 355; Hendrickx and Salgado-Barragán, 2002: 394; Ahyong, 2004: 526, fig. 168 a-c; Rodrigues and Young, 2005: 240; Almeida et al., 2007b: 7; Ahyong and Jarman, 2009: 93; Schram, 2010: 90.

Alimerichthus cylindricus Holthuis and Manning, 1964: 138.

Material examined: 1 specimen (AKA 14; 09007'20"S / 34053'40"W; 72 m) (MOUFPE 1002) September 1965; 1 specimen damaged (SALD A55) (MOUFPE 1003); 1 specimen (Tamandaré beach-PE; 08\%44'S / 3506'W; subtidal zone) (MOUFPE 1004); 2 specimens (SALD 1981; 13048.5S / 38048.5'W; $49 \mathrm{~m}$ ) (MOUFPE 1005) 23 September 1968; 1 specimen (AKA 59; 09०46'10"S / 35०34'40"W; $31 \mathrm{~m}$ ) (MOUFPE 1007) 07 September 1965; 1 specimen (Tambaú-PB; 0707'S / 3452'W; subtidal zone) (MOUFPE 1008) 06 September 1971; 4 specimens (SALD 1981B; 12059.7'S / 38०19.5'W; 59 m) (MOUFPE 1009) 27 September 1968; 1 specimen (CAN 91; 06²4'S / 3446'W; 54 m) (MOUFPE 1010) 21 December 1965; 1 specimen (AKA 32; 0924'S / 3514'W; 27 m) (MOUFPE 1012); 1 specimen (SALD 1648; 07030'S / 34º32'W; $37 \mathrm{~m}$ ) (MOUFPE 1013) 07 September 1967; 3 specimens (SALD 7566; 02016'S / 4115'48”W; $41 \mathrm{~m}$ ) (MOUFPE 1014) 20 July 1987; 1 specimen (SALD 7597; 02¹5'S / 41057'W; $47 \mathrm{~m}$ ) (MOUFPE 1015) 30 July 1987; 1 specimen (Forte Orange-PE 02; 07048'S / 34050'W; subtidal zone) (MOUFPE
1016) 08 April 1989; 1 specimen (Cond./ Suape 15; 08²1'S / 34057'W) (MOUFPE 1017) 20 July 1978; 1 specimen (SALD 7624; 0101'S / 4335'W; 53 m) (MOUFPE 1019) 12 August 1987; 1 specimen (CAN 50; 0351'S / 37042'W) (MOUFPE 1022); 4 specimens (GM 29; 00³8’ N / 4552.5’W; 75 m) (MOUFPE 1023) 06 June 1969; 1 specimen (Jaguaribe-PE; 07043'S / 34050'W; subtidal zone) (MOUFPE 1024) 02 October 1971; 1 specimen (SALD 1649; 07026'S / 34030'W; $51 \mathrm{~m}$ ) (MOUFPE 1025) 06 October 1967; 1 specimen (PE 36; 07040'S / 34²8'W; 60-88 m) (MOUFPE 1026) 14 February 1969; 3 specimens (SALD 1743A; 0051'S / 43041.4'W; $75 \mathrm{~m}$ ) (MOUFPE 1027) 04 November 1967; 3 specimens (SALD 1684A; 03059.5'S / 35०53.7'W; $140 \mathrm{~m}$ ) (MOUFPE 1028) 18 October 1967; 1 specimen (REC 149; 07056'S / 34037'W; 38 m) (MOUFPE 1029); 1 specimen (CAN 14; 01030'S / 38048'W) (MOUFPE 1031) July 1965; 2 specimens (SALD 1684; 03059.5'S / 35053.7'W; 75 m) (MOUFPE 1032) 18 October 1967; 1 specimen (Tambaú-PB; 0707'S / 34º52'W; subtidal zone) (MOUFPE 1033) 07 September 1971; 1 specimen damaged (SALD 2447; 02²4'N / 48²4'W; $85 \mathrm{~m}$ ) (MOUFPE 1034); 1 specimen (SALD 1711; 02²5'S / 39'30.5'W; 49 m) (MOUFPE 1035) 27 October 1967; 1 specimen (SALD 1732A; 02¹3'S / 41'51'W; 69 m) (MOUFPE 1037); 1 specimen (CAN 87; 0708'S / 34'26'W) (MOUFPE 1038) December 1965; 2 specimens (CAN 30; 02049'S / 38055'W) (MOUFPE 1039) August 1965; 1 specimen (CAN 18; 01056'S / 37051'W) (MOUFPE 1040); 1 specimen (CAN 31; 02052'S / 38०52'W; $72 \mathrm{~m}$ ) (MOUFPE 1041) 11 August 1965; 1 specimen (AKA-Mouth of São Francisco River; 10\%44'S 36²1'W) (MOUFPE 1042); 2 specimens (SALD 1655; 0604'S / 3459'W; 25 m) (MOUFPE 1043); 1 specimen (GM 153; 02¹6’N / 47049'W; 85 m) (MOUFPE 1045); 1 specimen (Tamandaré beach-PE-dredge; 0844'S / 3506'W; 90 m) (MOUFPE 1046) 24 May 1965; 3 specimens (SALD 1708; 02044'S / 3901.5'W; 65 m) (MOUFPE 1047) 22 October 1967; 1 
specimen damaged (SALD 1663A; 03049,6'S / 33049.2'W; $53 \mathrm{~m}$ ) (MOUFPE 1048) 09 October 1967; 1 specimen (SALD 1701A; 01057'S / 37046'W; 57 m) (MOUFPE 1049); 1 specimen (CAN 30; 02049'S / 38055'W) (MOUFPE 1050) August 1965; 1 specimen (GM 45; 0132'N / 47034’W; 67 m) (MOUFPE 1051) 08 June 1969; 1 specimen (CAN 101; 04048'S / 3506'W; 69 m) (MOUFPE 1052); 1 specimen (SALD 1738A; 01055.5'S / 42045'W; $75 \mathrm{~m}$ ) (MOUFPE 1053) 31 October $1967 ; 3$ specimens resected (CAN 89; 06046'S / 34\%36'W; 72 m) (MOUFPE 1054) 21 December 1965; 1 specimen (CAN 48; 04001'S / 37º33'W) (MOUFPE 1055) August 1965; 1 specimen (CAN 33; 02०58'S / 38\%45'W) (MOUFPE 1056) August 1965; 2 specimens (SALD 1711A; 02²5'S / 39॰30'W; 75 m) (MOUFPE 1057) 27 October 1967; 2 specimens (SALD 1767A; 01057.5’ N / 47013.5’W; $93 \mathrm{~m}$ ) (MOUFPE 1058) 13 November 1967; 2 specimens (SALD 1750; 0004'S / 44033.5'W; $52 \mathrm{~m}$ ) (MOUFPE 1059) 06 November 1967; 2 specimens damaged (CAN 13; 01030's / 38०48'W; 45-49 m) (MOUFPE 1060) 11 July 1965; 4 specimens (SALD 1729; 02¹0.5'S / 41027'W; $53 \mathrm{~m}$ ) (MOUFPE 1061) 30 October 1967; 1 specimen (CAN 107; 04040'S / 3606'W; 56 m) (MOUFPE 1062) 15 January 1966; 2 specimens (SALD 1647A; 07030'S / 34º34.7’ W; $37 \mathrm{~m}$ ) (MOUFPE 1063) 06 October 1967; 1 specimen (SALD 1684; 03059.5'S / 35053.7'W; $75 \mathrm{~m}$ ) (MOUFPE 1065) 18 October 1967; 3 specimens (CAN 52; 03040'S / 37054'W; $54 \mathrm{~m}$ ) (MOUFPE 1067) 24 August 1965; 1 specimen (Vila Velha-PE 6; 07048'S / 3451'W; subtidal zone) (MOUFPE 1068) 16 September 1989; 1 specimen damaged (CAN 74; 0358'S / 3556'W; 70 m) (MOUFPE 1153); 1 specimen (REVIZEENE I 210; 03050'17”S / 37037'05”W) (MOUFPE 12335) 12 October 1995; 1 specimen (REVIZEE-NE I 237; 07047'53"S / 34029'06"W; 52.89 m) (MOUFPE 12337) 17 October 1995; 1 specimen (REVIZEE-NE I 235; 07048'S / 33०34'05'W; 42 m) (MOUFPE 12338) October 1995; 1 specimen (REVIZEE-
NE I 93; 03059'35”S / 35055'23”W; 64 m) (MOUFPE 12339) October 1995; 1 specimen (REVIZEE-NE I 225; 06²1'47"S / 34045'54”W; $57 \mathrm{~m}$ ) (MOUFPE 12341) 14 October 1995; 1 specimen (REVIZEE-NE III 42; 0329'46”S / 3808'53”W; 19 m) (MOUFPE 12342) 16 May 1998; 1 specimen (REVIZEE-NE III 81A; 01\%43’48”S / 37॰07'16”W; 55 m) (MOUFPE 12343) 06 June 1998; 1 specimen (REVIZEE-NE III 40; 4'05'38"S / 37025'34"W; 50 m) (MOUFPE 12345) 15 May 1998; 1 specimen (REVIZEENE I 210; 0350'17”S / 37037'05”W) (MOUFPE 12512) 12 October 1995; 6 specimens (REVIZEE-NE III 33; 06050'42"S / 34039'W; 35 m) (MOUFPE 12557) 13 May 1998; 3 specimes (BPot IV 35-dredge; 04047.48'S / 3644.81'W; 19.7 m) (MOUFPE 14062) 28 May 2004; 1 specimen (BPot IV 30-dredge; 04047.46'S / 36³9.38'W; 22.5 m) (MOUFPE 14063) 28 May 2004; 2 specimens (BPot IV 38-dredge; 04047.50'S / 3650.22'W; $15 \mathrm{~m}$ ) (MOUFPE 14.064) 29 May 2004; 1 specimen (BPot IV 05-dredge; 04047.40'S / 36¹7.77’W; $85 \mathrm{~m}$ ) (MOUFPE 14069) 30 May 2004; 1 specimen (Bpot IV A10; 4048'38.40"S / 36042'15”W; $17 \mathrm{~m}$ ) (MOUFPE 14124) 20 May 2004; 1 specimen (Bpot III 02-dredge; 0458.21'S / 36²12.29'W; $10.1 \mathrm{~m}$ ) (MOUFPE 14131) 21 November 2003; 4 specimens (Bpot III 38-dredge; 04047.50'S / 36050.22'W; $11.1 \mathrm{~m}$ ) (MOUFPE 14132) 24 November 2003; 1 specimen (BPot III 41-dredge; 04º36.64'S / 36º55.83’W; 24.5 m) (MOUFPE 14133) 24 November 2003; 1 specimen (Bpot III 34-dredge; 04042.07'S / 36044.88'W; 26 m) (MOUFPE 14134) 24 November 2003; 1 specimen (BPot III 14-dredge; 4047.41's / 36²6.89'W; $27 \mathrm{~m}$ ) (MOUFPE 14135) 24 November 2003; 1 specimen (BPot III 17-dredge; 04057.67'S / 36²5.93'W; $11.2 \mathrm{~m}$ ) (MOUFPE 14136) 22 November 2003; 1 specimen (Bpot III 42-dredge; 04042'09”S / 36055.63'W; 20.2 m) (MOUFPE 14137) 24 November 2003; 2 specimens (BPot III 03-dredge; 04051.43'S / 36¹2.36'W; $29.1 \mathrm{~m}$ ) (MOUFPE 14138) 21 November 2003; 1 specimen (BPot III 38-Van Veen A; 04047.50'S / 3650.22'W; $11.1 \mathrm{~m}$ ) 
(MOUFPE 14155); 1 specimen (Bpot III 09; 0504.75'S / 36018.93'W; $1.6 \mathrm{~m}$ ) (MOUFPE 14184) 16 November 2003; 1 specimen (Bpot III MF 34; 501'03.9”S / 36²5’05.6”W; 7.1 m) (MOUFPE 14185) 22 November 2003; 1 specimen (BPot II 35B; 04047.48'S / 3644.81'W; $15.8 \mathrm{~m}$ ) (MOUFPE 14207) May 2003; 1 specimen (BPot II 13-dredge; 04047.41'S / 36²3.17'W; $47.1 \mathrm{~m}$ ) (MOUFPE 14208) 23 May 2003; 2 specimens (BPot II 39-dredge; 04042.08'S / 3650.23’W; $15.1 \mathrm{~m}$ ) (MOUFPE 14209) 19 May 2003; 1 specimen (BPot II 40-dredge; 04036.66'S / 36050.25'W; $79.1 \mathrm{~m}$ ) (MOUFPE 14210) 19 May 2003; 2 specimens (BPot II 03-dredge; 0451.43'S / 36¹2.36'W; $29.1 \mathrm{~m}$ ) (MOUFPE 14214) 22 May 2003; 7 specimens (BPot I 13B-dredge; 04⒋41'S / 36²3.17’W; $47.1 \mathrm{~m}$ ) (MOUFPE 14270) July-August 2002; 2 specimens resected (BPot I 13C-dredge; 04047.41'S / 36²3.17’W; $47.1 \mathrm{~m}$ ) (MOUFPE 14271) August 2002; 2 specimens (BPot I 14C-dredge; 4047.41'S / 36²6.89'W; 27 m) (MOUFPE 14272) August 2002; 1 specimen (Bpot I 21B-dredge with algae; 04058.30'S / 36033.94'W; 9.6 m) (MOUFPE 14273) August 2002; 1 specimen (BPot I 23C-dredge; 04047.42'S / 36³3.97'W; 21.8 m) (MOUFPE 14274) August 2002; 1 specimen (BPot II 24-dredge; 04041.09'S / 36³4.03'W; $67.1 \mathrm{~m}$ ) (MOUFPE 14275) 24 May 2003; 1 specimen (BPot I 30B-dredge; 04047.46'S / 36³9.38'W; $18.8 \mathrm{~m}$ ) (MOUFPE 14276) August 2002; 2 specimens (BPot I 35C-dredge; 04047.48'S / 36044.81'W; $15.8 \mathrm{~m}$ ) (MOUFPE 14277) August 2002; 1 specimen (Petrobrás 1 PEP 1-Control 2) (MOUFPE 14866) December 2003; 3 specimens (BPot-PAI 081 CES 41 Extra; 0300'17”S / 38०51'26”W; 55 m) (MOUFPE 14900) 07 July 2009; 1 specimen (BPot-PAI 121 RNS 42 Extra; 04046'58”S / 36²5'08”W; $45.7 \mathrm{~m}$ ) (MOUFPE 14901) 15 July 2009; 1 specimen (Paracurú-CE) (MOUFPE 14902) November 2003; 1 specimen (Poço do Cravo- 03 R1; 1324'S / 38²'W; $100 \mathrm{~m}$ ) (MOUFPE 14903) May 2009; 1 specimen (BPot-PAI 075 CES 23 Extra; 03॰08'34"S / 3852'01”W; 33 m) (MOUFPE 14904) 09 July 2009; 1 specimen
(BPot-PAI 121 RNS 41 Extra; 04046'42”S / 36'25'24”W; $46 \mathrm{~m}$ ) (MOUFPE 14905) 13 July 2009; 1 specimen (BPot-PAI 144 RRN 01-Extra; $04^{\circ} 19.1483^{\prime}$ S / 37²0.7081'W; 20 m) (MOUFPE 14906) 11 July 2009; 1 specimen (Petrobrás PAT $21500 \mathrm{~J}$ sample 2) (MOUFPE 14907) December 2003; 2 specimens (BPot-PAI 081 CES 42 Extra; 0300'08”S / 38०51'05”W; $59.7 \mathrm{~m}$ ) (MOUFPE 14908); 1 specimen (BPot-PAI 121 RNS 34 Extra; 04046'56”S / 36²5'32”W; 44.3 m) (MOUFPE 14909) 14 July 2009; 1 specimen (BPot-PAI 121 RNS 13 Extra; 04047'00”S / 36²5'24”W; 44 m) (MOUFPE 14910) 13 July 2009; 1 specimen (BPot-PAI 081 CES 41 Extra; 0300'17”S / 38051'26”W; $55 \mathrm{~m}$ ) (MOUFPE 14911) ; 2 specimens (BPotPAI 144 RRN 03 Extra; 04019'24"S / 37020'08”W; 20 m) (MOUFPE 14912) 12 July 2009; 2 specimens (BPot-PAI 081 CES 32 Extra; 0300'15”S / 38०51'08”W; 58 m) (MOUFPE 14913); 3 specimens (Bpot-PAI 144 RRN 05 Extra; 04¹9'30”S / 37020'51"W; $20 \mathrm{~m}$ ) (MOUFPE 14914) 12 July 2009; 1 specimen (BPot-PAI 081 CES 33 Extra; 0300'26”S / 38०51'04”W; 58 m) (MOUFPE 14915) 07 July 2009; 1 specimen (BPot-PAI 121 RNS 21 Extra; 04046’5 "'S / 36²5’24”W; 43 m) (MOUFPE 14916) 13 July 2009; 1 specimen (BPot-PAI 121 RNS 32 Extra; 04046'58”S / 36²5'16”W; 44 m) (MOUFPE 14917) 15 July 2009; 1 specimen (BPot-PAI 081 CES 44 Extra; 0300’38”S / 3851'17”W; 53 m) (MOUFPE 14918) 08 July 2009; 1 specimen (BPot-Regional MR 26 CO R2; 04048'13"S / 3654'53”W; 13 m) (MOUFPE 14964) 02 May 2010; 1 specimen (BpotRegional MR 32 R3 Extra; 04049'11"S / 36²5'32”W; $19 \mathrm{~m}$ ) (MOUFPE 14965) 27 October 2009.

Distribution: Western Atlantic Bermuda, Bahamas, Florida, Belize, British Honduras, Nicaragua, Panama, Cuba, Jamaica, Dominican Republic, Puerto Rico, Virgin Islands, Guadeloupe, St. Eustatius, Barbuda, Antigua, Colombia, Venezuela, Bonaire, Curaçao, Tobago, southward to Brazil (Rocas Atoll and Amapá to São Paulo). Central Atlantic - St. Helena. Eastern Atlantic 
- Cape Verde Islands, San Pedro Bay, St. Vincent, Senegal. Indo-West Pacific - Red Sea, Somalia, Arabian Sea, Persian Gulf, Sri Lanka, Seychelles, Comores, Mozambique, Madagascar, La Réunion, Mauritius, Thailand, Vietnam, Taiwan, Japan, South China Sea, Indonesia, Papua New Guinea, Philippines, Australia, Lord Howe Island, New Caledonia, Chesterfield Atoll, Loyalty Islands (Uvea), Tuamotu, Hiva Oa, Nuku Hiva, Marquesas, Chesterfield Islands. Eastern Pacific - Hawaii (24000’N / 167º00'W) (Schram, 2010).

Habitat: All kinds of bottom, from intertidal to $110 \mathrm{~m}$ depth (Manning, 1969). Material examined is from shallow subtidal to $140 \mathrm{~m}$.

Remarks: This species has a near pantropical distribution (Ahyong, 2001). The Brazilian specimens showed a slightly striped red pattern on telson, but this was not found in all specimens.

\section{Genus Pseudosquillisma Cappola and Manning, 1995 \\ Pseudosquillisma oculata (Brullé, 1837)}

Squilla oculata Brullé, 1837: 18, fig. 3. Squilla monodactyla A. Milne-Edwards, 1878: 232.

Pseudosquilla oculata-Miers, 1880: 110, pl. 3, figs. 3-4; Kemp, 1913: 102; Holthuis, 1941: 266; Lemos de Castro, 1955: 29, figs. 22-23, pl. 8 and16; Manning, 1969: 271, figs. 7576; 1977: 103 ; Coelho and Koening, 1972: 251; Manning and Chace, 1990: 71; GomesCorrêa, 1998: 295.

Pseudosquillisma oculata - Cappola and Manning, 1995: 285; Moosa, 2000: 408, tab. 1; Ahyong, 2001: 117, fig. 57; 2002: 369; Hendrickx and Salgado-Barragán, 2002: 394; Schram, 2010: 94.

Material examined: 1 specimen (Santo Aleixo Island; 08³6'S / 3502'W; subtidal zone) (MOUFPE 1169); 1 specimen (Candeias beach-PE; 08¹2'S / 34º56'W; subtidal zone) (MOUFPE 1170) 16 May 1905; 1 specimen (Suape Bay-PE; 08²2'S / 34º57’W; subtidal zone) (MOUFPE 1171) 07 August 1963.

Distribution: Western Atlantic - Florida;
Bahamas, Mexico, Nicaragua, Dominican Republic, Puerto Rico, Virgin Islands, Guadeloupe, Colombia, Brazil (Rio Grande do Norte to Espírito Santo). Central Atlantic - Ascension and St. Helena Islands. Eastern Atlantic - S. Thomé, Cape Verde Islands, Azores, Canary Islands, Portugal and Gulf of Guinea. Indo-West Pacific - Somalia, Madagascar, Mauritius, South China Sea, Indonesia, Australia, Samoa, French Polynesia, Hawaii, Easter Island (Rodrigues and Young, 2005).

Habitat: Usually found in reef habitats and under rocks, at depths from subtidal to 69 m (Schram, 2010).

Remarks: The specimens agree very well with the description given by Manning (1969) and Ahyong (2001).

\section{Superfamily Lysiosquilloidea Giesbrecht, 1910}

\section{Family Lysiosquillidae Giesbrecht, 1910}

Genus Lysiosquilla Dana, 1852

Lysiosquilla scabricauda (Lamarck, 1818)

Squilla scabricauda Lamarck, 1818: 188. Lysiosquilla inornata Dana, 1852: 615. Lysiosquilla maculata - Boone, 1930: 11, 29, pl. 3 [not L. maculata Fabricius, 1793]. Lysiosquilla scabricauda - Glassell, 1934: 454; Lemos de Castro, 1955: 34, fig. 24, pl. 10 and 17; Manning, 1967: 104; 1969: 24, figs. 2-4, 5a-b; 1977: 287; Coelho and Koening, 1972: 250, tab.; Camp, 1973: 10, fig. 2; Fausto Filho, 1978: 66, tab. I; Manning and Camp, 1993: 89, fig. 3d-f.; Gomes-Corrêa, 1998: 292; Ahyong, 2001: 130; Hendrickx and SalgadoBarragán, 2002: 394; Schram, 2010: 111.

Material examined: 1 specimen (Tamandaré Bay-PE; 0844'S / 3506’W; subtidal zone) (MOUFPE 1154) 07 June 1905; 1 specimen (Raposa beach-MA; 02²4'S / 44005'W; subtidal zone) (MOUFPE 1155) 26 September 1978; 2 specimens (Tamandaré Bay-PE; 0844'S / 3506'W; subtidal zone) (MOUFPE 1221) 1967; 1 specimen (Itamaracá Island-PE; 07048'S / 34050'W; subtidal zone) (MOUFPE 14956) June 2000. 
Distribution: Western Atlantic Bermuda, Mississippi, Louisiana, Texas, Bahamas, North Carolina, Florida, Honduras, Nicaragua, Mexico, Panama, Antigua, Colombia, Venezuela, Trinidad, Brazil (Piauí to Santa Catarina) and numerous records in Manning (1969: 25-27).

Habitat: In soft bottoms burrows, at depths from shallow subtidal to $200 \mathrm{~m}$ (Schram, 2010).

Remarks: Dana (1852) did not select a type species for Lysiosquilla but $L$. inornata (= L. scabricauda) was selected as the type of the genus by Fowler (1912). Manning (1961) found designated a syntype of $L$. inornata as lectotype. The specimens agree very well with the description given by Manning (1969).

Genus Lysiosquillina Manning, 1995 Lysiosquillina glabriuscula (Lamarck, 1818)

Squilla glabriuscula Lamarck, 1818: 188.

Squille glabriscule - H. Milne Edwards, 1837: 519.

Squilla vittata H. Milne Edwards, 1837: 519. Lysiosquilla glabriuscula - Miers, 1880: 7; Holthuis, 1941: 36; Lemos de Castro, 1955: 37, fig. 25, 43e, 54, pl. 11, 17; Manning, 1969: 34, figs. 5c-d, 6; Coelho and Koening, 1972: 250, tab.; Fausto Filho, 1978: 66, tab. I; Schotte and Manning, 1993: 571, fig. 2; Gomes-Corrêa, 1998: 292.

Lysiosquillina glabriuscula - Manning, 1995: 133; Ahyong, 2001: 137; Hendrickx and Salgado-Barragán, 2002: 394; Schram, 2010: 113.

Material examined: 1 specimen (CAN. 13; 0130'S / 3848'W; 45 m) (MOUFPE 1212) 11 July 1965; 1 specimen (BPot-PAI 121 RNS 33 Extra; 04047’07”S / 36²5'24”W; $40 \mathrm{~m}$ ) (MOUFPE 14895) 14 July 2009; 1 specimen (Candeias beach-PE; 0811'S / 34054'W; subtidal zone) (MOUFPE 14983) 17 November 2007.

Distribution: Western Atlantic - North and South Carolina, Georgia, Florida, Mexico, Bahamas, Jamaica, Honduras, Dominican Republic, Puerto Rico, St. Vincent, Virgin Islands, Guadeloupe, Colombia, Tobago,
Brazil (Ceará to São Paulo) (Gomes-Corrêa, 1998).

Habitat: In coral reefs and sandy bottoms, on depths from shallow subtidal to $50 \mathrm{~m}$ (Gomes-Corrêa, 1998; Schotte and Manning, 1993).

Remarks: The specimens agree very well with the description given by Manning (1969). The color was almost faded, but the dark band pattern cited by Schotte and Maninng (1993) was preserved.

\section{Family Nannosquillidae Manning, 1980}

Genus Alachosquilla Schotte and Manning, 1993

Alachosquilla floridensis (Manning, 1962)

Lysiosquilla floridensis Manning, 1962: 221. Acanthosquilla floridensis - Rodrigues, 1966: 142; Manning, 1969: 67, fig. 16; Coelho and Koening, 1972: 250, tab.

Alachosquilla floridensis - Schotte and Manning, 1993: 573, fig. 3; Schram, 2010: 120.

Material examined: Holotype (Cape Florida, Key Biscayne, Florida, USA) (USNM 107875); 1 specimen (Piedade beach-PE; 08¹0'S / 34056'W; subtidal zone) (MOUFPE 1274) 12 September 1962; 1 specimen (Petrobrás1 PEP 1-500 M; 0308'07”S / 3848'43”W; $37 \mathrm{~m}$ ) (MOUFPE 14862) December 2001; 1 specimen (BPot-PAI 075 CES 41 R1 (0-10); 0308'45”S / 38०52'05”W; 35 m) (MOUFPE 14938) 09 July 2009; 1 specimen (BPot-PAI 075 CES 42 R1 (0-10); 0308'31"S / 38०52'17"W; 35 m) (MOUFPE 14939) 08 July 2009.

Distribution: Western Atlantic - Florida, Virgin Islands, Tobago, Venezuela to Brazil (Bahia and São Paulo) (Schotte and Manning, 1993). Northern distribution filled with records from Ceará and Pernambuco States.

Habitat: In coral reefs, rocky walls and burrows on sandy substrates, at depths from shallow subtidal to $28 \mathrm{~m}$ (Schotte and Manning, 1993). The deepest bathymetric limit is increased to $37 \mathrm{~m}$. 
Remarks: The specimens analysed are in agreement with Manning's (1969) description. The mandibular palp was absent and the fused ocular scale showing a square shape.

Genus Bigelowina Schotte and Manning, 1993

Bigelowina biminiensis (Bigelow, 1893)

Lysiosquilla biminiensis Bigelow, 1893: 102. Acanthosquilla biminiensis - Holthuis, 1967: 3; Manning, 1969: 63, figs. 14-15; Reaka and Manning, 1980: 11; Gomes-Corrêa, 1998: 292.

Bigelowina biminiensis - Schotte and Manning, 1993: 574, fig. 4; Schram, 2010: 123.

Material examined: Lectotype (Nixies' Harbor, South Bimini) (USNM 17999); 1 specimen (BPot-PAI 121 RRN 3 R1 (010); 04051'38”S / 35057'15”W; $46.5 \mathrm{~m}$ ) (MOUFPE 14940) 15/07/2009; 1 specimen (BPot-PAI 121 RRN 1 R3; 04051'21"S / 35०57'53"W; $46.7 \mathrm{~m}$ ) (MOUFPE 14941) 15 July 2009; 1 specimen damaged (BPotPAI 121 RNS 23 R2 (0-10); 04047'02"S / 36²5'24"W; $42.7 \mathrm{~m}$ ) (MOUFPE 14942) 14 July 2009; 1 specimen (BPot-PAI 121 RNS 44 R1 (0-10); 04046'58”S / 36²5'40”W; 43.2 m) (MOUFPE 14943) 14 July 2009; 1 specimen (BPot-PAI 121 RRN 1 Extra; 04051'21"S / 35०57'53”W; $47 \mathrm{~m}$ ) (MOUFPE 14944) 15 July 2009.

Distribution: Western Atlantic Bahamas, North Carolina, Georgia, Texas, Cuba, Tobago, Colombia, Brazil (Rio Grande do Norte and São Paulo). Eastern Pacific Costa Rica, Panama (Manning, 1974). The species is herein recorded from Rio Grande do Norte State.

Habitat: On sand, rocks and in coral reefs, shallow to $46 \mathrm{~m}$ depths (Schotte and Manning, 1993).

Remarks: Manning (1969) selected the most intact male from syntypes as lectotype. The specimens agree with the lectotype identified by Bigelow (1893). Manning (1969) incorrectly reported fused ocular scales in $B$. biminiensis; the ocular scales in the present series are separate with a bilobed shape, as in the lectotype. The mandibular palp is present as cited by Manning (1969).

\section{Bigelowina spp.}

Material examined: 1 specimen juvenile (BPot-PAI 144 RRN 1 Extra; 04019'09"S / 37020'43”W; $20 \mathrm{~m}$ ) (MOUFPE 14945) 11 July 2009; 1 specimen (BPot-PAI 075 CES 31 Extra; 0308'40”S / 38०52'03”W; 36 m) (MOUFPE 14946) 09 July 2009; 1 specimen juvenile (BPot-PAI 121 RRN 1 (0-10); 04'51'21"S / 35०57'53”W; 47 m) (MOUFPE 14947) 15 July 2009; 1 specimen (BPotPAI 075 CES 44 R2 (0-10); 0308'43”S / 38०51'47”W; $35 \mathrm{~m}$ ) (MOUFPE 14948) 09 july 2009.

Remarks: These specimens are juveniles or damaged, so species identification was not possible.

Genus Nannosquilla Manning, 1963

Nannosquilla dacostai Manning, 1970

Nannosquilla dacostai Manning, 1970: 101, fig. 2 - Schotte and Manning, 1993: 567; GomesCorrêa, 1998: 293; Schram, 2010: 129.

Material examined: Holotype (Camocim, Ceará, Brazil; 02³1'S / 40²2'W; $23 \mathrm{~m}$ ) (USNM 128332); 1 specimen (NE II 112/111; 3,838 $\mathrm{O}$ / 33,687\%W; $28 \mathrm{~m}$ ) (MOUFPE 12333) 03 March 1997; 1 specimen (Bpot-PAI 121 RNS 12 R1; 04046'58”S / 36²5'23"W; 44 m) (MOUFPE 14897) 14 July 2009.

Distribution: Western Atlantic Brazil (Ceará) (Manning, 1970). Southern distribution increased with records from Rio Grande do Norte and Fernando de Noronha Chain-PE (this study).

Habitat: From 23 to $44 \mathrm{~m}$ depth.

Remarks: Manning (1970) described the median prominence flanked laterally by obtusely rounded lateral projection, but the specimens analysed in this study do not present this feature. Otherwise the characters agree well with the original description. 
Nannosquilla potiguara Albuquerque and Coelho, 2009

Nannosquilla potiguara Albuquerque and Coelho, 2009: 56, fig.1.

Material examined: Holotype (Bpot II 06; 04º52.84'S / 36º $17.83^{\prime} \mathrm{W} ; 16.9$ m) (MOUFPE 14022) 22 May 2003; 1 paratype (Bpot III E14; 04059.94'S / 36²6.80'W; water depth: $8.8 \mathrm{~m}$ ) (MOUFPE 14154) 15 November 2003; 1 specimen (BPot-PAI 075 RCE 5 R2; 0328'17”S / 38¹5’38”W; 37 m) (MOUFPE 14898) 10 July 2009.

Distribution: Only known from the type locality, Rio Grande do Norte State, Brazil (Albuquerque and Coelho, 2009).

Habitat: On sandy bottoms, at depths from 8 to $37 \mathrm{~m}$.

Remarks: This is the first record of the species after the original description, but no morphological variations were found between them. Color has faded and could not be recognized.

\section{Superfamily Squilloidea Latreille, 1802 Family Squillidae Latreille, 1802 Genus Cloridopsis Manning, 1968 Cloridopsis dubia (H. Milne Edwards, 1837)}

Squilla dubia H. Milne Edwards, 1837: 522. Squilla rubrolineata Dana, 1852: 618. Squilla desaussurei Stimpson, 1857: 503. Lysiosquilla desaussurei - Miers, 1880: 8. Squilla alba-Boone, 1930: 35, pl. 5 [non $S$. alba Bigelow, 1893].

Squilla dubia - Lemos de Castro, 1955; Manning, 1967: 13, fig. 9, pl. II, fig. 34e, pl. XIII, fig. 45.

Cloridopsis dubia - Manning, 1969: 141; Coelho and Koening, 1972: 250, tab.; Reaka and Manning, 1980: 15; Gomes-Corrêa, 1998: 291; Boyko, 2000: 49; Bento and Melo, 2010: 148; Schram, 2010: 179.

Material examined: 1 specimen (Vila Velha-ITA 6; 07048'S / 34051'W; subtidal zone) (MOUFPE 1161) 08 March 1989; 1 specimen (Sobrado-Itamaracá 3; 07048'S / 34051'W; subtidal zone) (MOUFPE 1162) 26 August 1988; 4 specimens (Santa Cruz
Channel-ITA; 07048'S / 34051'W; intertidal zone) (MOUFPE 1163) 08 October 1991; 1 specimen (Vila Velha, Itamaracá Island-PE; 07048'S / 3451'W; subtidal zone) (MOUFPE 1200) 06 April 1974; 4 specimens (Vila VelhaSanta Cruz channel-PE; 07048'S / 34051'W; intertidal zone) (MOUFPE 1201) 12 July 1973; 1 specimen (Santa Cruz channel; 07048'S / 34051'W; intertidal zone) (MOUFPE 1202) 17 March 1972; 1 specimen damaged (GaibúPE; 08 18'S / 34056'W; subtidal zone) (MOUFPE 1203) 27 June 1993; 1 specimen (Santa Cruz channel; 07048'S / 34051'W; intertidal zone) (MOUFPE 1204) 22 March 1967; 1 specimen (FURENE 112-Vila Velha; 07048'S / 3451'W; subtidal zone) (MOUFPE 1205) 21 November 1972; 1 specimen (Vila Velha-Santa Cruz channel; 07048'S / 34051'W; subtidal zone) (MOUFPE 1206) 23 October 1971; 2 specimens (São José do Ribamar - MA; 02³3'48”S / 4403'12”W; subtidal zone) (MOUFPE 1208) 01 July 1970; 1 specimen (Itamaracá Island; 07048'S / 34051'W; subtidal zone) (MOUFPE 1209) 22 September 1972; 1 specimen (Genipapeiro; 02'31'S / 441'' W; subtidal zone) (MOUFPE 1210) 09 November 1983.

Distribution: Western Atlantic - South Carolina, Georgia, Mexico, Guatemala, Dominican Republic, British Honduras, Cuba, Trinidad, Panama, Colombia, Venezuela, Brazil (Pará to Santa Catarina). Eastern Pacific - Mexico, El Salvador, entrance of Panama Canal, Colombia, Peru (Manning, 1969; Bento and Melo, 2010).

Habitat. Usually on shallow mud flats; salt lake, mud and mouth of river at depths of 0 to $73 \mathrm{~m}$ (Reaka and Manning, 1980).

Remarks: The specimens agree well with description of Manning (1969). This species is reported from estuarine habitats, with mud flat substrate (Manning, 1969).

Genus Meiosquilla Manning, 1968

Meiosquilla quadridens (Bigelow, 1893)

Squilla quadridens Bigelow, 1893: 102 Manning, 1961: 14, pl. 3, figs. 1-2.

Meiosquilla quadridens - Manning, 1969: 
106, figs. 31, 33a; Camp, 1971: 125; Coelho and Koening, 1972: 250, tab.; Camp, 1973: 18, fig. 6, tab. 4; Holmquist et al., 1989: 255, tab. 2.; Gomes-Corrêa, 1998: 291; Bento and Melo, 2010: 150; Schram, 2010: 196.

Material examined: Holotype (Key Largo, Florida, USA; ALBATROSS 2604; $\left.25^{\circ} 05^{\prime} \mathrm{N} / 80^{\circ} 15^{\prime} \mathrm{W} ; 102 \mathrm{~m}\right)$ (USNM 11547) 09 April 1886; 2 specimens (REVIZEENE I 213; 4039'43"S / 3643'23”W; 52 m) (MOUFPE 12585) 1995; 1 specimen (BPot IV 40 Van Veen A; 04036.66'S / 3650.25'W; $78 \mathrm{~m}$ ) (MOUFPE 14070) 29 May 2004; 1 specimen (BPot IV 05-dredge; 04047.40'S / 36¹7.77'W; $85 \mathrm{~m}$ ) (MOUFPE 14072) 30 May 2004; 1 specimen (BPot IV 24-dredge; 04041.09'S / 36³4.03'W; 72 m) (MOUFPE 14076) 24 May 2004; 1 specimen (BPot III 40 Van Veen B; 04036.66'S / 3650.25'W; 83.1 m) (MOUFPE 14153) 25 November 2003; 1 specimen (BPot II 13-dredge; 04047.41'S / 36²3.17'W; $47.1 \mathrm{~m}$ ) (MOUFPE 14202) 23 May 2003; 1 specimen (BPot II 05-dredge; 04º47.40'S / 36º17.77'W; 100 m) (MOUFPE 14203) 24 May 2003; 1 specimen (BPot II 14-dredge; 04047.41'S / 36²6.89’W; 27 m) (MOUFPE 14204) 23 May 2003; 1 specimen (Manati OC8 R2; 1331'S / 3849'W; 45 m) (MOUFPE 14937) 15 February 2009.

Distribution: Western Atlantic - North Carolina, Georgia, Florida, Gulf of Mexico, Bahamas, Colombia, off Venezuela, British Guiana, Surinam, Brazil (Maranhão, Rio Grande do Norte to Pernambuco and Rio de Janeiro) (Bento and Melo, 2010).

Habitat: On coral gravel bottoms, at depths from littoral to $137 \mathrm{~m}$ (Manning, 1969).

Meiosquilla schmitti (Lemos de Castro, 1955)

Squilla quadridens - Holthuis, 1941: 32.

Squilla schmitti Lemos de Castro, 1955: 8, figs. 5-8, pl. 1, figs. 32-33; - Manning, 1961: 17, pl. 3, figs. 3-4.

Meiosquilla schmitti - Manning, 1969: 111, figs. 32, 33b; Coelho and Koening, 1972: 250, tab.; Camp, 1973: 24, fig. 8, tab. 5; Fausto Filho and Sampaio Neto, 1976: 65; Gomes-
Corrêa, 1998: 291; Schram, 2010: 197.

Material examined: 1 specimen (REC 148; 07052'S / 34º32'W; 46 m) (MOUFPE 1173); 3 specimens (SALD 1843; 0546'S / 3501.4'W; $47 \mathrm{~m}$ ) (MOUFPE 1174) 13 April 1968; 1 specimen (SALD 1872; 0120'S / 4333.5'W; 50 m) (MOUFPE 1175) 23 April 1968; 1 specimen (SALD 1760A; 01010.7’ N / 46032.5'W; $100 \mathrm{~m}$ ) (MOUFPE 1176) 08 November 1967; 3 specimens (SALD 1767; $01^{\circ} 32.5^{\prime} \mathrm{N} / 47^{\circ} 24.5^{\prime} \mathrm{W} ; 63 \mathrm{~m}$ ) (MOUFPE 1177) 13 November 1967; 1 specimen damaged (SALD 1755; 00³7.5'S / 4538.5'W; $80 \mathrm{~m}$ ) (MOUFPE 1178) 07 November 1967; 3 specimens (SALD 1875; 00056'S / 43041.5'W; 49 m) (MOUFPE 1179) 23 April 1968; 1 specimen (Fortaleza-CE; 03043'S / 38³0'W; subtidal zone) (MOUFPE 1180) 04 July 1965; 1 specimen damaged (GM. 116; $02^{\circ} 57.5^{\prime} \mathrm{N} / 4^{\circ} 45^{\prime} \mathrm{W} ; 81 \mathrm{~m}$ ) (MOUFPE 1181) 13 September $1970 ; 2$ specimens (SALD 2447; 02²4'N / 48²4'W; 85 m) (MOUFPE 1182); 1 specimen (SALD 1675A; 0511'S / 3509.7'W; $33 \mathrm{~m}$ ) (MOUFPE 1183) 16 October 1967; 1 specimen (SALD 1817; 02¹2.7'S / 42०25.5'W; $60 \mathrm{~m}$ ) (MOUFPE 1184) 28 November 1967; 4 specimens (SALD 1718; 0207'S / 40³6'W; $71 \mathrm{~m}$ ) (MOUFPE 1185) 28 October $1967 ; 1$ specimen (SALD 1739A; 02¹4'S / 42॰43'W; 35 m) (MOUFPE 1186) 31 October 1967 ; 1 specimen damaged (SALD 1749A; 0003'N / 44032.3’W; 63 m) (MOUFPE 1187) 06 November 1967; 1 specimen damaged (GM. II 186; $04^{\circ} 12.5^{\prime} \mathrm{N} /$ 50¹2'W; $84 \mathrm{~m}$ ) (MOUFPE 1188); 1 specimen damaged (GM. 183; 043'N / 50³5'W; 84 m) (MOUFPE 1190); 3 specimens (SALD 1813; 0129'S / 4319'W; 83 m) (MOUFPE 1191) 28 November 1967; 3 specimens (SALD 1750; 0004'S / 44³3.5'W; 52 m) (MOUFPE 1192) 06 November 1967; 1 specimen (Suape-PE; 08²2'S / 34\%57’W; subtidal zone) (MOUFPE 1193) 25 September 1965; 1 specimen (SALD PAVASAS 03-dredge; 02॰46'S / 39॰30'W; 25 m) (MOUFPE 9060) 19 July $1987 ; 1$ specimen (REVIZEE-NE I 225; 06021'47”S / 34045'54”W; 57 m) (MOUFPE 12336) 14 October 1995; 1 specimen (REVIZEE-NE I 213; 4039'43"S / 
36043'23”W; $52 \mathrm{~m}$ ); 2 specimens (REVIZEENE III 180; 1202'31"S / 37014'24”W) (MOUFPE 12587) 19 July 1998; 1 specimen (BPot IV 03-dredge; 04051.43'S / 36¹2.36'W; $33.5 \mathrm{~m}$ ) (MOUFPE 14073) 26 May 2004; 1 specimen (BPot IV 24-dredge; 04041.09'S / 36³4.03'W; $72 \mathrm{~m}$ ) (MOUFPE 14075) 24 May 2004; 1 specimen (BPot IV 42-dredge; 04042'09S / 3655.63'W; 23.5 m) (MOUFPE 14121) 29 May 2004; 1 specimen (BPot IV 34-dredge; 04042.07'S / 3644.88'W; $27.5 \mathrm{~m}$ ) (MOUFPE 14122) 29 May 2004; 1 specimen (BPot IV 23-dredge; 04047.42'S / 36³3.97’W; $21.8 \mathrm{~m}$ ) (MOUFPE 14123) 27 May 2004; 1 specimen (BPot III 41-dredge; 04036.64'S / 36055.83'W; $24.5 \mathrm{~m}$ ) (MOUFPE 14147) 24 November 2003; 1 specimen (BPot III 31 Van Veen A; 04042.05'S / 36³9.43'W; 38.5 m) (MOUFPE 14148) 24 November 2003; 1 specimen (BPot III 30-dredge; 04047.46'S / 36039.38'W; $18 \mathrm{~m}$ ) (MOUFPE 14149) 23 November 2003; 1 specimen (Bpot III 42-dredge; 04042'09”S / 36055.63'W; 20.2 m) (MOUFPE 14150) 24 November 2003; 9 specimens (Bpot III 03-dredge; 04051.43'S / 36¹2.36'W; $29.1 \mathrm{~m}$ ) (MOUFPE 14151) 21 November 2003; 1 specimen (BPot III 28-dredge; 04056.43'S / 36²1.35W; 14.3 m) (MOUFPE 14152) 23 November 2003; 1 specimen (BPot III 31 Van Veen C; 04042.05'S / 36³9.43'W; $38.5 \mathrm{~m}$ ) (MOUFPE 14156) 24 November 2003; 4 specimens (BPot II 03-dredge; 04051.43'S / 36º 12.36'W; $29.1 \mathrm{~m}$ ) (MOUFPE 14205) 22 May 2003; 1 specimen (BPot I 03A; 04051.43'S / 36²12.36'W; $29.1 \mathrm{~m}$ ) (MOUFPE 14278) August 2002; 1 specimen (BPot I 07B-dredge; 04058.23'S / 36¹7.73'W; $10.5 \mathrm{~m}$ ) (MOUFPE 14279) August 2002; 1 specimen (BPot I 23A-dredge; 04047.42'S / 36³3.97'W; 21.8 m) (MOUFPE 14280) August 2002; 1 specimen (Petrobrás PCR 1-1000M; 0305'43.3”S / 3846'57.5W; $47 \mathrm{~m}$ ) (MOUFPE 14865) December 2003; 1 specimen (BCAM OC 5B; 1329'S / 38०49' W; $45 \mathrm{~m}$ ) (MOUFPE 14867) 19 January 2008; 1 specimen (BCAM-40 OC 8 R3; 13031'S / 3849'W; 45 m) (MOUFPE 14919) May 2007; 1 specimen (OC 04 R2; 1327'S / 38०49'W; $45 \mathrm{~m}$ ) (MOUFPE 14920); 2 specimens
(Manati CO1 R4; 1322'S / 3855’W; 50 m) (MOUFPE 14921) 16 January 2009; 2 specimens (ES RRN 6 R2 (2-5); 20²6'S / 39052'W; slope) (MOUFPE 14922) October 2010; 1 specimen (Petrobrás OC 10B; 1329'S / 38º51'W; 40 m) (MOUFPE 14923) 20 January 2008; 1 specimen (Manati OC 4 R1; 1327'S / 38०49'W; 45 m) (MOUFPE 14924) 16 February 2009; 3 specimens (Manati PMNT R1) (MOUFPE 14925) 15 February 2009; 1 specimen (Poço do Cravo CO 03 R3; 1324'S / 38\%42'W) (MOUFPE 14926) May 2009; 1 specimen (Manati PMNT R2) (MOUFPE 14927) 15 February 2009; 1 specimen (BPot-PAI 075 CES 34 Extra; 0308'40”S / 3851'55”W; $34.5 \mathrm{~m}$ ) (MOUFPE 14928) 09 July 2009; 1 specimen (BPot-PAI 121 RNS 11 Extra; 04046'57”S / 36²5'24”W; $45 \mathrm{~m}$ ) (MOUFPE 14929) 13 July 2009; 1 specimen (BPot-PAI 075 CES 24 Extra; 0308'39"S / 38051'59”W; 34 m) (MOUFPE 14930) 08 July 2009; 3 specimens (BPot-PAI 121 RRN 4 Extra; 04052'02"S / 35०57'36”W; $40 \mathrm{~m}$ ) (MOUFPE 14931) 15 July 2009; 2 specimens (BPot-PAI 121 RRN 5 Extra; 04051'45”S / 35०58'02”W; 41.6 m) (MOUFPE 14932) 15 July 2009; 1 specimen (BPot-PAI 081 CES 11 R3 (0-10); 03000'22"S / 38051'13”W; 56 m) (MOUFPE 14933) 07 July 2009; 1 specimen (BPot-PAI 121 RNS 44 R3; 0446.8943'S / 36²5.6745'W; 42.5 m) (MOUFPE 14934) 14 July 2009; 1 specimen (BPot-PAI 081 CES 42-Extra; 0300.1320'S / 3851.0849’W; $59.7 \mathrm{~m}$ ) (MOUFPE 14935) 07 July 2009; 2 specimens (Bpot-PAI 075 CES 21 Extra; 0308'39"S / 38०52’03”W; 36 m) (MOUFPE 14936) 09 July 2009; 1 specimen (BPot-Regional MR 32 R1; 04049'11"S / 36²5'32”W; 22 m) (MOUFPE 14967) 27 October 2009; 1 specimen (BPot-Regional MR 44 R3; 04038'28"S / 36'45'37”W; $50 \mathrm{~m}$ ) (MOUFPE 14968) 23 October 2009; 2 specimens (BPot-Regional MR 44 R1; 04³8'28”S / 36045'37"W; 50 m ) (MOUFPE 14969) 23 October 2009; 2 specimens (BPot-Regional MR $41 \mathrm{CL}$ R1; 04049'40”S / 36¹0'06”W; 59 m) (MOUFPE 14970) 2010; 1 specimen (Bpot-Regional MR 34 R1; 04051'35”S / 36³9'18”W; 13 m) 
(MOUFPE 14971) 22 May 2010; 1 specimen (BPot-Regional MR 42 R2; 04045'54"S / 36²5'49”W; $51 \mathrm{~m}$ ) (MOUFPE 14972) 25 October 2009; 1 specimen (BPot-Regional MR 32 R2 Extra; 04049'11"S / 36²5'32”W; 19 m) (MOUFPE 14973) 27 October 2009; 1 specimen (BPot-Regional MR 33 R1; 04048'58”S / 36³6'10”W; 24 m) (MOUFPE 14974) 22 May 2010.

Distribution: Western Atlantic Florida, Gulf of Mexico, Bahamas, Colombia, Venezuela, Brazil (Abrolhos Archipelago and Amapá to Rio de Janeiro) (Gomes-Corrêa, 1998).

Habitat: In coral gravel, sandy and mud bottoms, the depth range is from shallow sublittoral to $100 \mathrm{~m}$ depth (Coelho and Koening, 1972).

Remarks: The specimens agree well with diagnosis and description given by Manning (1969). Some dark spaced chromatophores were found on specimens, mainly on the telson.

\section{Meiosquilla tricarinata (Holthuis, 1941)}

Squilla tricarinata Holthuis, 1941: 32-35, fig. 1.

Meiosquilla tricarinata - Manning, 1969: 114, figs. 33c, 34; Coelho and Koening, 1972: 250, tab.; Gomes-Corrêa, 1998: 291; Bento and Melo, 2010: 150; Schram, 2010: 198.

Material examined: 1 specimen (REVIZEE-NE III 93; 3045'04”S / 32०19'58”W; $70 \mathrm{~m}$ ) (MOUFPE 12558) 02 June 1998; 1 specimen juvenile (BPot II 34 Van Veen A; 0442.07'S / 3644.88'W; 7.1 m) (MOUFPE 14206) 20 May 2003.

Distribution: Western Atlantic - Florida (off Jensen beach, Indian River region), Bahamas, Virgin Islands, Lesser Antilles, Colombia (Northeast of Santa Marta: Punta Aguja), Aruba, Brazil (Fernando de Noronha and Abrolhos Archipelagos and Pernambuco, Bahia and Espírito Santo) (Bento and Melo, 2010). Herein reported to Rio Grande do Norte State, extending its northern distribution in Brazil.

Habitat: In coral gravel, at depths from subtidal to $48 \mathrm{~m}$ (Manning, 1969), extended herein to $70 \mathrm{~m}$.

\section{Meiosquilla spp.}

Material examined: 1 specimen damaged (PMNT R1; absent data) (MOUFPE 14861); 1 specimen (BPot-Regional MR 41 CL R2; 04049'40"S / 36¹0'06"W; 58 m) (MOUFPE 14975); 27 October 2009.

Remarks: These specimens are juveniles or damaged and identification to species level is not possible.

\section{Genus Alima Leach, 1817 \\ Alima hildebrandi (Schmitt, 1940)}

Squilla hildebrandi Schmitt, 1940: 152, fig. 6. Squilla empusa Balss, 1916: 50 [part, non Squilla empusa Say, 1818].

Squilla labadiensis Ingle, 1960: 566, figs. 1-10. Alima hieroglyphica - Manning, 1969: 135, fig. 40; 1977: 112, figs. 36, 51; Coelho and Koening, 1972: 250, tab.; Gomes-Corrêa, 1998: 291; Almeida et al. 2007a: 8.

Squilla hieroglyphica - Ortiz and Silva, 1992: 12.

Alima hildebrandi - Ahyong, 2001: 188; Almeida et al. 2008: 25; Schram, 2010: 205.

Material examined: Holotype (Fort Sherman, Canal Zone, Panama) (USNM 76068) 03 March 1937; 1 specimen (Santo Aleixo Island-PE-trawling; 08³6'35”S / $35^{\circ} 01^{\prime} 27^{\prime \prime} \mathrm{W} ; 1 \mathrm{~m}$ ) (MOUFPE 13432) 04 November 2005.

Distribution: Western Atlantic from Panama, Cuba, and Brazil (Ceará to Pernambuco, Bahia and São Paulo). Eastern Atlantic - from off West Africa: Senegal (Gorée), Ivory Coast, Ghana, Congo (Almeida et al., 2007a, as A. hieroglyphica).

Habitat: On fine quartz muddy sand, at depths from the intertidal to $20 \mathrm{~m}$ (Manning, 1977).

Remarks: Alima hildebrandi was removed from the synonymy of $A$. hieroglyphica sensu stricto by Ahyong (2001) who considered both species as valid. Alima hildebrandi presents a telson with prelateral lobe and is restricted 
to the Atlantic, while A. hieroglyphica has no prelateral lobe and is restricted to the IndoWest Pacific (for more details see Ahyong, 2001).

Alima neptuni (Linnaeus, 1768)

Cancer neptuni Linnaeus, 1768: 226. Holthuis, 2000: 17.

Alima hyalina Leach, 1817: appendix 4.

Alima angusta Dana, 1852: 631.

Squilla alba Bigelow, 1894: 539, pl. 22 Hendrickx and Salgado-Barragán, 2002: 395.

Squilla hyalina-Manning, 1967: 105.

Alima hyalina-Manning 1962: 496; Manning, 1969: 128, figs. 37-39; 1970: 1432, fig. 1; 1977: 115, figs. 37, 51; Coelho and Koening, 1972: 250, tab.; Fausto Filho and Sampaio Neto, 1976: 66.

Alima alba - Schotte and Manning, 1993: 577.

Alima neptuni - Manning and Lewinsohn, 1986: 13; Ahyong, 2001: 188; 2002: 395; Schram, 2010: 206.

Material examined: Neotype (Bimini, Bahamas Islands) (USNM 18495); 1 specimen (REVIZEE-NE III 28; 07²7'11'S / 34031'30”W; 43 m) (MOUFPE 12591) 10 May 1998; 1 specimen (BPot-PAI 075 CES 14 Extra; 0308'38”S / 38²'01”W; 34.7 m) (MOUFPE 14957) 08 July 2009; 1 specimen (BPot-PAI 075 CES 11 R2; 0308'37”S / 38०52'02”W; 33.7 m) (MOUFPE 14958) 09 July 2009; 1 specimen (BPot-PAI 121 RNS 13 R2 (0-10); 04047'00”S / 36²5'24”W; 42 m) (MOUFPE 14959) 14 July 2009; 1 specimen (BPot-PAI 144 RNS 44 Extra; 04039'24”S / 36053'04”W; $22 \mathrm{~m}$ ) (MOUFPE 14960) 13 July 2009; 1 specimen (BPot-PAI 075 CES 13 R1 (0-10); 0308'36”S / 38²5'02”W; 34 m) (MOUFPE 14961) 09 July 2009 (Fig. 2).

Distribution: Western Atlantic - Gulf of Mexico, Bahamas, Florida (Peanut Island in Lake Worth, Palm Beach County), Tobago (Buccoo Reef), Dominican Republic, Barbuda, Curaçao, Colombia (Manning, 1969). From Ceará to Paraíba State, extending its southern distribution in Atlantic (this study). Eastern - Cape Verde Islands. Central Atlantic - St.
Helena. Indo-Pacific - Red Sea, South Africa, Madagascar Great Reef, Tuléar, Indonesia (Kongsi Island), Marquesas, Hawaii (Waikiki Reef, Honolulu) (Manning, 1969).

Habitat. Back reef area on sandy patches and tidal flats exposed at low tide, from intertidal to $70 \mathrm{~m}$ (Schotte and Manning, 1993).

Remarks: Alima neptuni was described based on a larva of $A$. hyalina (Leach, 1817), which lead to a huge confusion when the adult forms were analysed (see Ahyong, 2001; Schotte and Manning, 1993). Manning (1962) studying larval development, synonymized A. alba with $A$. hyalina and Ahyong (2001) placed $A$. hyalina as junior synonym of $A$. neptuni, based on presence of two rounded lobes between terminal spines on uropodal protopod. Because the larval holotype of $A$. neptuni was lost and the description given by Linnaeus (1768) insufficient, Holthuis (2000) selected the lectotype of Squilla alba Bigelow, 1894 as the neotype of $A$. neptuni, making the two species objective synonyms, to fix the identity of the species. Alima neptuni is part of the genus group (with $A$. pacifica Ahyong, 2001) that share the following characters: six teeth on the dactylus of the raptorial claw, sharp telson denticles in adults and the uropodal protopod bears two lobes between the terminal spines (for more details, see Ahyong, 2001; 2012). The specimens examined herein agree well with description by Ahyong (2001).

\section{Genus Gibbesia Manning and Heard, 1997} Gibbesia neglecta (Gibbes, 1850)

Squilla neglecta Gibbes, 1850: 200.

Chloridella neglecta - Lunz, 1937: 154, fig. 4. Squilla neglecta - Lemos de Castro, 1955: 22, fig. 17, pl. 6-38, pl. 15-49; Manning, 1969: 181, figs. 50b, 51; Coelho and Koening, 1972: 250, tab.; Camp, 1973: 37, fig. 13, tab. 9; Gomes-Corrêa, 1998: 290.

Gibbesia neglecta - Manning and Heard, 1997: 313, fig. 14; Almeida et al., 2007a: 7; 2008b: 6; Bento et al., 2008: 62, figs. 1a, b; Schram, 2010: 236.

Material examined: 1 specimen damaged 

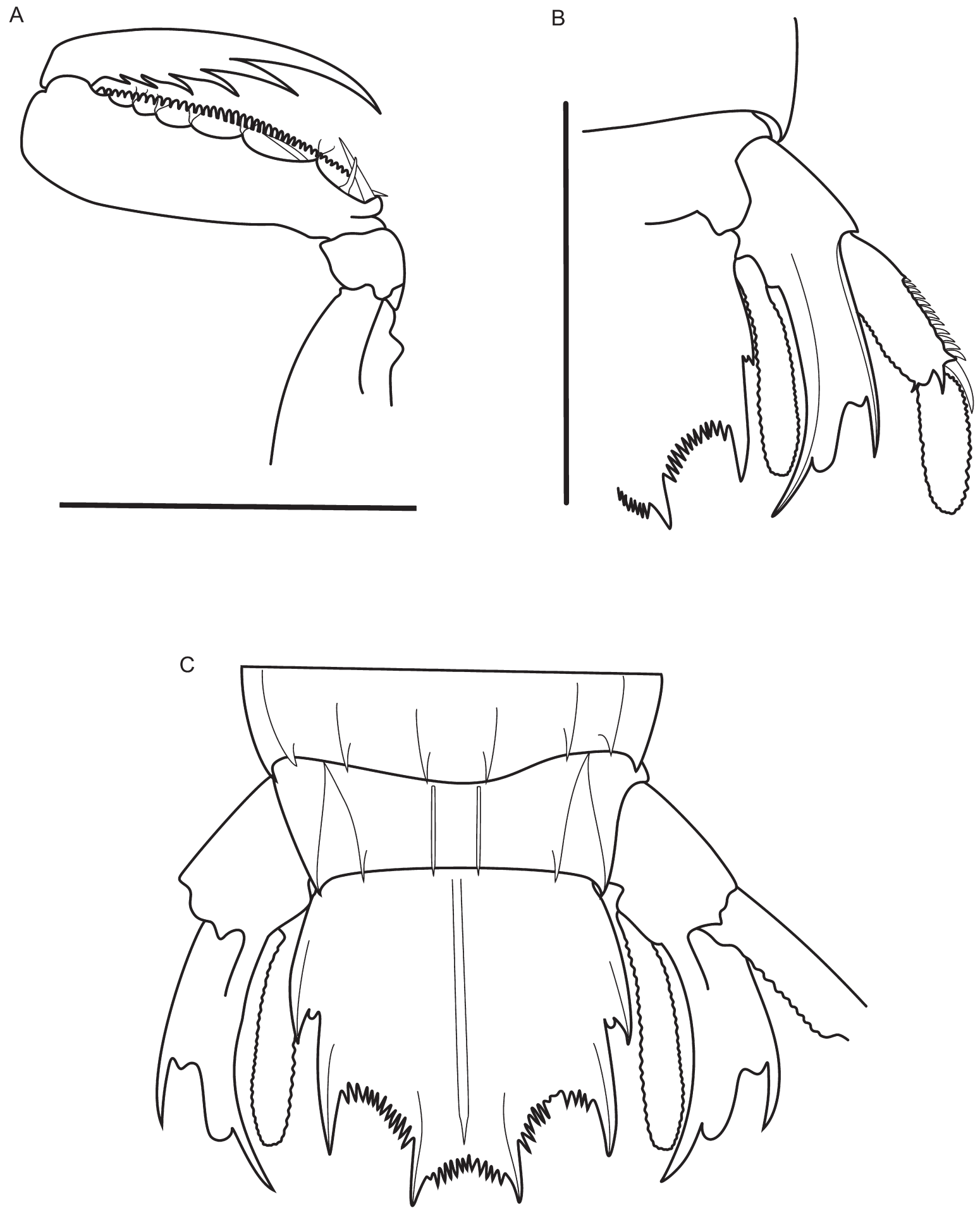

Figure 2. Alima neptuni (Linnaeus, 1768), female MOUFPE 14961; Bacia Potiguar-PAI 075 CES 13 R1 (0-10); 0308'36”S 38052'02”W). (A) Right raptorial claw, ventral view. (B) Left uropod, ventral view. (C) Telson, dorsal view. Scale bar $=0.5 \mathrm{~cm}$. 
(AKA mouth of São Francisco River; 1044'S / 36²1'W) (MOUFPE 1165) 01 December 1965; 1 specimen (ITA 93; 07035'S / 34047'W; 9 m) (MOUFPE 1166); 1 specimen (SALD 1765A; 00³1'N / 47049'W; 39 m) (MOUFPE 1167) 1212 November 1967; 1 specimen (AKA-mouth of São Francisco River; 1044'S / 36²1'W) (MOUFPE 1199) 01 December 1965; 1 specimen (BPot II MF 22; 502'00.3”S / 36²1409.4”W; $8.4 \mathrm{~m}$ ) (MOUFPE 14211) 29 May 2003.

Distribution: Western Atlantic - North Carolina to Jupiter Inlet, Florida, Gulf of Mexico from West Florida to Texas, Brazil (Ceará to Rio Grande do Sul) (Gomes-Corrêa, 1998).

Habitat: Muddy and sandy bottoms with detritus, from littoral to $540 \mathrm{~m}$ depth (Bento et al., 2008).

\section{Gibbesia prasinolineata (Dana, 1852)}

Squilla prasinolineata Dana, 1852: 630 - Lemos de Castro, 1955: 15, figs. 12-15, pl. 3; 35, pl. 13-46; Manning, 1967: 105; 1969: 175, figs. 49, 50a; 1977: 292; Coelho and Koening, 1972: 250, tab.; Gomes-Corrêa, 1998: 250, tab; Hendrickx and Salgado-Barragán, 2002: 395.

Gibbesia prasinolineata - Ahyong, 2001: 184; Bento et al., 2008: 63, fig. 1c, d; Schram, 2010: 236.

Material examined: 1 specimen (TutóiaMA; 02046'S 42\%16'W; subtidal zone) (MOUFPE 1168) 23 May 1978; 1 specimen damaged (Barra de Almofala-CE; 02050'S 4008'W; subtidal zone) (MOUFPE 1214) 27 January 1967.

Distribution: Western Atlantic - Florida, Mexico, Cuba, Jamaica, Virgin Islands (St. Thomas), Brazil (Pará to Santa Catarina) (Gomes-Corrêa, 1998).

Habitat: On muddy bottoms from shallow water to $75 \mathrm{~m}$ depth (Bento et al., 2008).

Genus Squilla Fabricius, 1787

Squilla brasiliensis Calman, 1917

Squilla brasiliensis Calman, 1917: 139, figs.
1-3 - Lemos de Castro, 1955: 18-22, fig. 16A, pl. 4, fig. 30, pl. 5, fig. 37, pl. 14, figs. 47-48; Manning, 1969: 215, figs. 57b, 60, 61a-b; Coelho and Koening, 1972: 250, tab.; GomesCorrêa, 1998: 289; Schram, 2010: 240.

Material examined: 1 specimen (SALD 2235A; 32०11'S / 5057.5W; 64 m) (MOUFPE 1157) 27 October 1969; 1 specimen (SUL II 11; 23044'S / 42०51'W; $184 \mathrm{~m}$ ) (MOUFPE 1158) 27 March 1972; 1 specimen (SALD 2259 - Uruguay; 3432.5'S / 530.' W; 60 m) (MOUFPE 1159) 09 November 1969.

Distribution: Western Atlantic - Brazil (Rio de Janeiro to Rio Grande do Sul) and Uruguay (many records in Manning, 1969: 215-216).

Habitat: At depths from 10 to $295 \mathrm{~m}$ (Gomes-Corrêa, 1998).

Squilla empusa Say, 1818

(Fig. 3)

Squilla empusa Say, 1818: 250 - Lunz, 1937: 8; Manning, 1961: 20, pl. 4, figs. 4-5; 1969 : 201, figs. 57a, 58-59; 1970: 257; Coelho and Koening, 1972: 250, tab.; Camp, 1973: 39, fig. 14, tab. 10; Hernández Aguilera and Hermoso Salazar, 1988: 2, fig. 1, tab. 1; Viana et al., 1998: 100; Hendrickx and SalgadoBarragán, 2002: 395; Bento and Melo, 2010: 150; Schram, 2010: 243.

Chloridella empusa - Fowler, 1912: 303; Rathbun, 1935: 119.

Material examined: 1 specimen damaged (SALD 1891; 013'N / 48²8'W; 24 m) (MOUFPE 6793) 01 May 1968 (Fig. 3).

Distribution: Western Atlantic - State of Maine (USA), Bermuda, Massachusetts, Rhode Island, Connecticut, New York, Long Island Sound, New Jersey, Delaware, Maryland, Chesapeake Bay, Virginia, North and South Carolina, Georgia, Florida, Gulf of Mexico, Alabama, Mississippi, Mexico (Campeche Bay), Nicaragua, Cuba, Jamaica, Trinidad, Colombia (Gulf of Urabá), Venezuela, Suriname (off mouths of rivers), French Guiana, numerous records in Manning (1969: 202-209), Brazil (Pará) (Viana et al., 1998). 

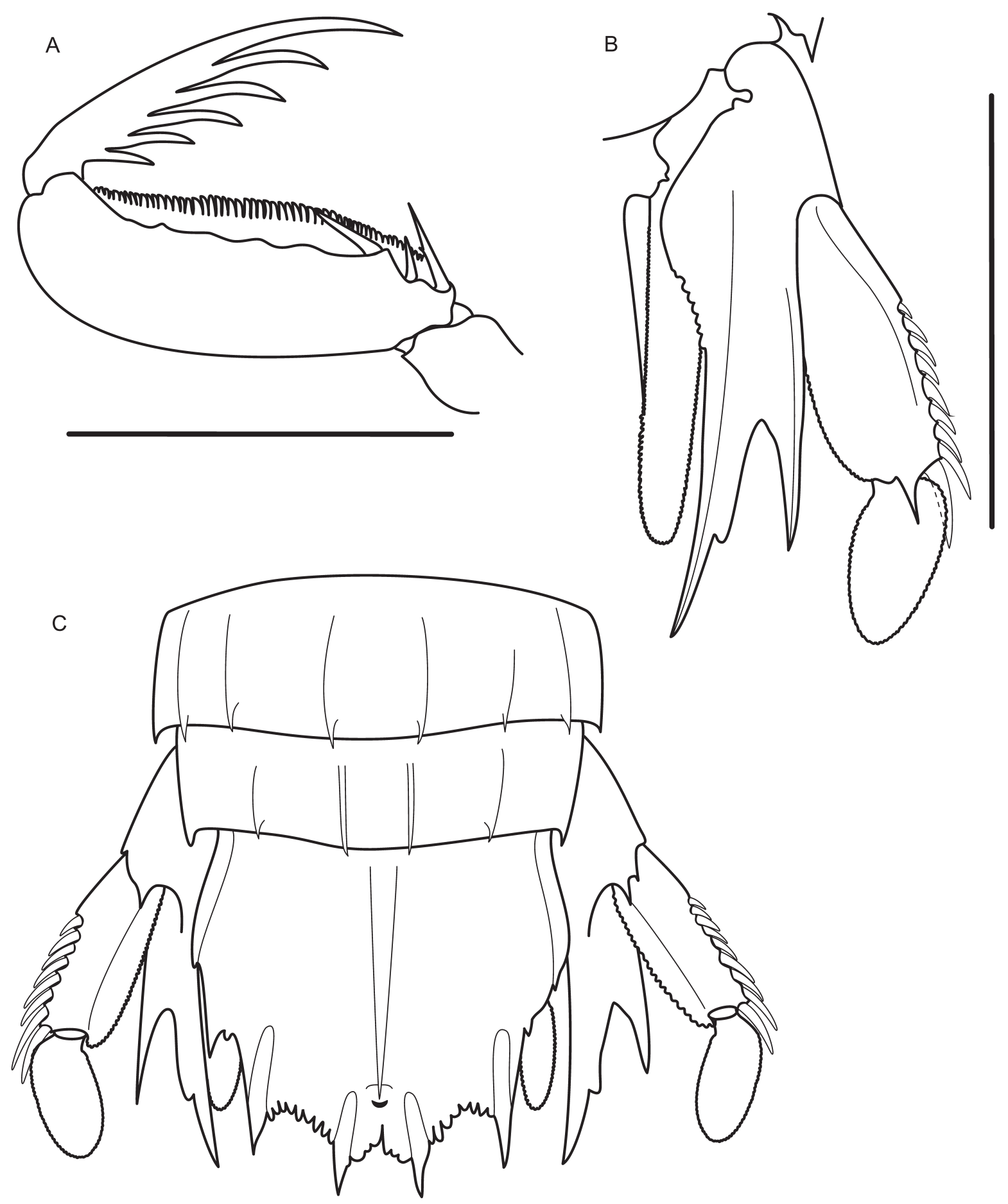

Figure 3. Squilla empusa Say, 1818, female MOUFPE 6793 (Almirante Saldanha 1891; 01`33’N 48²8’W; 24 m) 01 May 1968. (A aptorial claw, ventral view. (B) Left uropod, ventral view. (C) Telson, dorsal view. Scale bar $=0.5 \mathrm{~cm}$. 
Habitat: On silty-clay bottoms; in waters of low salinity. Depth range, 0-154 m (generally <40 m) (Manning, 1969).

Squilla grenadensis Manning, 1969

Squilla grenadensis Manning, 1969: 152, fig. 42 - Camp, 1973: 26, fig. 9, tab. 6; Fausto Filho, 1975: 133, fig. 1; Gomes-Corrêa, 1998: 290; Rodrigues and Young, 2005: 239, fig. 4; Bento and Melo, 2010: 150, fig. 3; Schram, 2010: 244.

Material examined: 1 specimen (SALD 2168; 2305.7'S / 4055.5'W; 103 m) (MOUFPE 1213) 19 September 1969; 1 specimen (GM III 15; 00²9.5'S / 43055'W; 110 m) (MOUFPE 1298); 1 specimen (BPot-PAI 121 RRN 3 Extra; 04051'38"S / 35०57'15”W) (MOUFPE 14868); 1 specimen (BPot-Regional MR 42 R2; 04045'54"S / 36025'49”W) (MOUFPE 14966).

Distribution: Western Atlantic Grenada, Gulf of Mexico, Brazil (Maranhão, Bahia and Santa Catarina) (Gomes-Corrêa, 1998; Bento and Melo, 2010). Herein reported to Rio Grande do Norte and Rio de Janeiro States.

Habitat: It is found on sandy and gravel bottoms on depth range between 54.9-311 m (Rodrigues and Young, 2005).

Squilla lijdingi Holthuis, 1959

Squilla lijdingi Holthuis, 1959: 181, fig. 76bd, pl. 9, figs. 1-2 - Manning, 1961: 26, pl. 6, figs. 1-3; 1969: 192, figs. 53b, 55; Fausto Filho, 1966: 139, fig. 1; Coelho and Koening, 1972: 250, tab.; Gomes-Corrêa, 1998: 290; Schram, 2010: 245.

Material examined: 1 specimen (SALD 1793B; 04013.5'N / 50²6’W; 75 m) (MOUFPE 1215) 18 November 1967; 2 specimens (GM. 179; 043'N / 510ㅜ' W; $63 \mathrm{~m}$ ) (MOUFPE 1216) 1971; 1 specimen (SALD 1892 II; 0145’ N / 48¹8'W; 56 m) (MOUFPE 1218) 01 May 1968.

Distribution: Western Atlantic Colombia (Dept. Guajira), Venezuela, Trinidad, Tobago, Guyanas, off Surinam $\left(6^{\circ} 24.5^{\prime} \mathrm{N} 5^{\circ} 03^{\prime} \mathrm{W}\right)$, Brazil (mouth of
Amazon River, Amapá to Pará) (GomêsCorrêa, 1998).

Habitat. On sandy, muddy and shell bottoms; often in mouths of rivers, in a depth range of 9-182 $\mathrm{m}$ (most often $<100 \mathrm{~m}$ ) (Manning, 1969).

\section{Squilla obtusa Holthuis, 1959}

Squilla obtusa Holthuis, 1959: 186, fig. 76 h-j, pl. 9, figs. 3-4 - Manning, 1961: 28, pl. 7, figs. 1-3.; 1969: 187, figs. 53a, 54; Coelho and Koening, 1972: 250, tab.; Gomes-Corrêa, 1998: 290; Rodrigues and Young, 2005: 238, fig. 3; Almeida et al. 2007a: 8; 2007b: 6; Schram, 2010: 247.

Squilla intermedia - Bigelow, 1901: 159 [not S. intermedia Bigelow, 1893].

Material examined: 1 specimen (AKA 179; 10³5'42”S / 36¹5’10”W; $64 \mathrm{~m}$ ) (MOUFPE 1194) 04 December 1965; 1 specimen damaged (SALD 1787A; 0331.5'N / 50¹1'W; 75 m) (MOUFPE 1195) 17 November 1967; 2 specimens (AKA Mouth of São Francisco River; 1044'S / 36²1'W; subtidal zone) (MOUFPE 1196); 1 specimen (Cachoeira River-BA; 14049'S / 3902'W; estuary zone) (MOUFPE 5385) 29 July 1999.

Distribution: Western Atlantic Colombia, Venezuela, Curaçao, Puerto Rico, Trinidad, Surinam, Brazil (Piauí to Espírito Santo) (Rodrigues and Young, 2005).

Habitat: On muddy and shell bottoms, in a depth range of 13-182 $\mathrm{m}$ (Manning, 1969).

Remarks: The specimens agree well with the diagnostic characters recognized by Manning (1969) and Gomes-Corrêa (1986). Some color features are given by Rodrigues and Young (2005).

Squilla surinamica Holthuis, 1959

Squilla surinamica Holthuis, 1959: 184, figs. 76e-g, pl. 8, fig. 5, pl. 9, fig. 5 - Manning, 1969: 185, figs. 48b, 52; Coelho and Koening, 1972: 250, tab.; Fausto Filho and Sampaio Neto, 1976: 65; Gomes-Corrêa, 1998: 290; Schram, 2010: 249. 
Material examined: 2 specimens (GM. 159; 0039.5'N / 4713'W; 43 m) (MOUFPE 1222) September 1970; 1 specimen (SALD 2002 II; 0320'N / 50¹1'W; 28 m) (MOUFPE 1223) 25 November 1968; 1 specimen (SALD 1785 II; 0302'N / 49²7’30”W; 45 m) (MOUFPE 1224) 16 November 1968; 1 specimen damaged (SALD 2454; 03047’ N / 50॰44'W) (MOUFPE 1225); 2 specimens (SALD 2010; 0341'N / 50³1'W; 30 m) (MOUFPE 1226) 27 November 1968; 1 specimen (GM. 177; 04011'30”N / 50॰40'W; $32 \mathrm{~m}$ ) (MOUFPE 1227) June 1971; 2 specimens damaged (GM. 161; 014' N / 48¹0'30"W; $47 \mathrm{~m}$ ) (MOUFPE 1228); 2 specimens (SALD 1992A II; 01⒉'N / 48³2'30"W; 19 m) (MOUFPE 1231) 23 November 1968; 3 specimens (GM.III 157; 01046'N / 47014'W; 77 m) (MOUFPE 10205) September 1970.

Distribution: Western Atlantic - French Guiana, Surinam, Venezuela, Brazil (Amapá, Pará and Pernambuco) (Gomes-Corrêa, 1998).

Habitat: On muddy bottoms, often in mouths of rivers (Manning, 1969), in a depth range of $15-77 \mathrm{~m}$.

\section{Superfamily Eurysquilloidea Ahyong and Harling, 2000 \\ Family Eurysquillidae Manning, 1977 \\ Genus Eurysquilla Manning, 1963 \\ Eurysquilla plumata (Bigelow, 1901)}

Lysiosquilla plumata Bigelow, 1901: 156, figs. 6-9.

Pseudosquilla plumata - Manning, 1959: 18 [list]; 1961: 3.

Eurysquilla plumata - Manning, 1966: 378, fig. 8c-e; 1969: 251, fig. 70; Camp, 1971: 125; Coelho and Koening, 1972: 251, tab.; Camp, 1973: 44, fig. 16, tab. 12; GomesCorrêa, 1998: 294; Schram, 2010: 151.

Material examined: Holotype (Mayaguez Harbor, Puerto Rico) (USNM 64823); 2 specimens (REVIZEE-NE III 184; 12056.95'S / 38¹3.59'W) (MOUFPE 12891) 20 July 1998; 1 specimen (PE 07 R3; 08040’S /
34053'W; 35 m) (MOUFPE 14899).

Distribution: Western Atlantic - Gulf of Mexico, Florida (Indian River Region: off Sebastian Inlet, Florida Keys, Puerto Rico, Colombia (Bahía de Santa Marta), Brazil (Abrolhos Island and Bahia) (Gomes-Corrêa, 1998). From now registered to Pernambuco State, extending its northern distribution in Brazil (this study).

Habitat: It is found on sandy bottoms, between 10 and $60 \mathrm{~m}$ depth.

\section{Eurysquilla petronioi Lucatelli, Ahyong, Bezerra and Santos, 2012}

Eurysquilla petronioi Lucatelli, Ahyong, Bezerra and Santos, 2012: 1, fig. 1.

Material examined: Holotype (Barbados, Caribbean Sea, Station NR1-3; $13^{\circ} 00^{\prime} \mathrm{N} /$ $59^{\circ} 30^{\prime} \mathrm{W} ; 91.5$ - $366 \mathrm{~m}$ ) (USNM 111111) 1960; 1 Paratype (Rio Grande do Norte, Brazil; BPot II No. 33, dredge; $04^{\circ} 36.64^{\prime} \mathrm{S} /$ $36^{\circ} 44.89^{\prime} \mathrm{W} ; 368 \mathrm{~m}$ ) (MOUFPE 14201) 20 May 2003.

Distribution: Presently known only from Barbados and Rio Grande do Norte, Brazil (Lucatelli et al., 2012).

Habitat: It is found on sandy muddy substrate, between 91.5 and $368 \mathrm{~m}$ depth (Lucatelli et al., 2012).

\section{Conclusions}

This paper records new distribution ranges for nine species. Squilla grenadensis was registered further north than the previously known occurrence in Rio Grande do Norte. The southernmost distribution limit was also increased for Neogonodactylus moraisi (to Rio Grande do Norte), Nannosquilla dacostai (to Rio Grande do Norte and Pernambuco). In other cases, the distribution gaps were filled to the north: Alachosquilla floridensis (to Rio Grande do Norte and Pernambuco), Bigelowina biminiensis (to Rio Grande do Norte), Eurysquilla plumata (to Pernambuco), and Meiosquilla tricarinata (to Rio Grande do 
Norte). Alima neptuni (Rio Grande do Norte and Paraíba) was recorded for the first time from Brazilian waters.

Furthermore, the collection contains some rare species, such as Nannosquilla dacostai Manning, 1970, Neogonodactylus moraisi (Fausto Filho and Lemos de Castro, 1973) and Neogonodactylus minutus (Manning, 1969), which are endemic to Brazilian coast. Accordingly, there are now 42 species recorded for Brazil of which 33 are deposited in the MOUFPE collection. New records and new species should be found as additional samplings are made unraveling the biodiversity of these important but cryptic benthic animals.

\section{Acknowledgements}

We thank Dr. Jesser F. Souza-Filho (Museu de Oceanografia da UFPE, Brazil), M. Sc. Catarina L. Araújo-Silva (Universidade do Porto, Portugal) for several comments and M.Sc. Marina S. L. C. Araújo (Museu de Oceanografia da UFPE, Brazil) for English review. The first author also thanks Dr. Petrônio A. Coelho, who passed away last November, for his contribution during his scientific and personal life. The first author also thanks the Coordenação de Aperfeiçoamento de Pessoal de Nível Superior (CAPES) for financial support and for the provison of a travel grant to visit collections abroad. Special thanks to Dr. Rafael Lemaitre (USNM) and Dr. Karen Reed (USNM) for the facilities provided to the first author for the revision of Stomatopoda species deposited in the collection at the Smithsonian Institution, Washington DC, and to anonymous reviewers for their suggestions on the text.

\section{References}

Ahyong, S.T. 2001. Revision of the Australian stomatopod Crustacea. Records of the Australian Museum, Supplement 26: 1-326.

Ahyong, S.T. 2002. Stomatopoda (Crustacea) from the Marquesas Islands: results of
MUSORSTOM 9. Zoosystema, 24: 347372.

Ahyong, S.T. 2004. Stomatopoda - mantis shrimps. p. 517-548. In: G. Poore (ed), Marine Decapod Crustacea of Southern Australia. Collingwood, CSIRO Publishing. Ahyong, S.T. 2005. Phylogenetic analysis of the Squilloidea (Crustacea: Stomatopoda). Invertebrate Systematics, 19: 189-208.

Ahyong, S.T. 2012. The marine fauna of New Zealand: mantis shrimps (Crustacea: Stomatopoda). NIWA Biodiversity Memoir, 125: 1-112.

Ahyong, S.T.; Chan, T.Y. and Liao, Y.C. 2008. A Catalog of the Mantis Shrimps (Stomatopoda) of Taiwan. Taipei: National Science Council, Taiwan, R.O.C, 190p.

Ahyong, S.T. and Harling, C. 2000. The phylogeny of the stomatopod Crustacea. Australian Journal of Zoology, 48: 607-642.

Ahyong, S.T, and Jarman, S.N. 2009. Stomatopod interrelationships: preliminary results based on analysis of three molecular loci. Arthropod Systematics and Phylogeny. 67: 91-98.

Ahyong, S.T.; Lowry, J.K.; Alonso, M.; Bamber, R.N.; Boxshall, G.A.; Castro, P.; Gerken, S.; Karaman, G.S.; Goy, J.W.; Jones, D.S.; Meland, K.; Rogers, D.C. and Svavarsson J. 2011. Subphylum Crustacea Brünnich, 1772. In: Zhang, Z.-Q. (Ed.) Animal biodiversity: An outline of higherlevel classification and survey of taxonomic richness. Zootaxa, 3148: 1-237.

Ahyong, S.T. and Norrington, S.F. 1997. Stomatopod Crustacea of the Maclaey Museum, University of Sydney. Proceedings of the Linnean Society of New South Wales, 118: 97-110.

Albuquerque, D.L. and Coelho, P.A. 2009. A new species of Nannosquilla (Stomatopoda: Nannosquillidae) from the Western Atlantic Ocean. Zootaxa, 2289: 55-60.

Almeida, A.O.; Bezerra, L.E.A.; SouzaFilho, J.F.; Almeida, S.M.; Albuquerque, D.L. and Coelho, P.A. 2008. Decapod and stomatopod crustaceans from Santo Aleixo Island, state of Pernambuco, Brazil. Nauplius, 16(1): 23-41. 
Almeida, A.O.; Coelho, P.A.; Santos, J.T.A. and Ferraz, N.R. 2007a. Crustáceos estomatópodos e decápodos da costa de Ilhéus, Bahia, Brasil. Atlântica, 29(1): 5-20.

Almeida, A.O.; Guerrazzi, M.C. and Coelho, P.A. 2007b. Stomatopod and decapod crustaceans from Camamu Bay, state of Bahia, Brazil. Zootaxa, 1553: 1-45.

Balss, H. 1916. Crustacea III: Stomatopoda, pp. 49-52. In: W. Michaelsen, Beiträge zur Kenntnis der Meeresfauna Westafrikas 2. Herausgegeben von W. Michaelsen.

Barreto, A.V., Calado, T.C.S.; Soares, C.M.A. and Araújo, J.S. 2003. Stomtopoda and Decapoda Anomura along the northeastBrazilian continental shelf and slope. Tropical Oceanography, 31(1): 63-69.

Bento, R.T.; Dall'Occo, P.L and Melo, G.A.S. 2008. On the diagnostic characters of the genus Gibbesia (Crustacea: Stomatopoda: Squillidae). Zootaxa, 1939: 61-64.

Bento, R.T. and Melo, G.A.S. 2010. Morphological considerations and range extensions for species of the superfamily Squilloidea (Crustacea: Stomatopoda) in the southwest Atlantic. Pan-American Journal of Aquatic Sciences, 5(1): 147-152

Bigelow, R.P. 1893. Preliminary notes on the Stomatopoda of the Albatross collections and on other specimens in the National Museum. Johns Hopkins University Circulars, 12: 100-102.

Bigelow, R.P. 1894. Report on the Crustacea of the order Stomatopoda collected by the steamer Albatross between 1885 and 1891 and on other specimens in the U.S. National Museum. Proccedings of United States National Museum, 17: 489-550, pls. 20-22.

Bigelow, R.P. 1901. The Stomatopoda of Porto Rico. Bulletin of the United States Fish Commission, 20(2): 149-160.

Boone, L. 1930. Crustacea: Stomatopoda and Brachyura. Scientific results of the cruises of the yachts "Eagle" and "Ara", 1921-1928, Wm. K. Vanderbilt, commanding. Bulletin of the Vanderbilt Marine Museum 2:1-228, pls. 1-74.

Borradaile, L.A. 1900. On the Stomatopoda and Macrura brought by Dr. Willey from the South Seas. p. 395-428, pls. 36-39. In: Zoological results based on material from New Britain, New Guinea, Loyalty Islands and elsewhere, collected during the years 1895, 1896, and 1897 (A. Willey ed.).

Boyko, C.B. 2000. The rise and fall of Lysiosquilla desaussurei and description of $L$. manningi n. sp.: the tale of the type. Journal of Crustacean Biology, 20 (special number 2): 48-55.

Brullé, M. 1837-1839. Crustacés. In: BarkerWebb, P. \& S. Berthelot, Histoire naturelle des les Canaries. Zoologie, 2: 2, Entomologie, 13-18, atlas (1837).

Calman W.T. 1917. Crustacea. Part 4. Stomatopoda, Cumacea, Phylocarida and Cladocera. British Antarctic ("Terra Nova”) Expedition, 1910. Natural History Report, Zoology, 3(5): 137-162.

Camp, D.K. 1971 Platysquilla horologii (Stomatopoda, Lysiosquillidae), a new species from the Gulf of Mexico, with an emendation of the generic definition. Proceedings of the Biological Society of Washington, 84: 119-127.

Camp, D.K. 1973. Stomatopod Crustacea. Memoirs of the Hourglass Cruises, 3(2): 1-92.

Cappola, V. and Manning, R.B. 1995. Research on the Coast of Somalia. Crustacea Stomatopoda. Tropical Zoology, 7: 271-291. Chopra, B. 1939. Stomatopoda. Scientific Reports John Murray Expedition, 6: 137-181. Coelho Filho, P.A. 2006. Checklist of the Decapods (Crustacea) from the outer continental shelf and seamounts from Northeast of Brazil REVIZEE Program (NE III). Zootaxa, 1184: 1-27.

Coelho P.A.; Almeida, A.O. and Bezerra, L.E.A. 2008. Checklist of the marine and estuarine Brachyura (Crustacea: Decapoda) of northern and northeastern Brazil. Zootaxa, 1956: 1-58.

Coelho, P.A. and Koening, M.L. 1972. Distribuição dos crustáceos pertencentes às ordens Stomatopoda, Tanaidacea e Isopoda no Norte e Nordeste do Brasil. Trabalhos Oceanográficos da Universidade Federal de Pernambuco, 13: 5-21. 
Coelho, P.A. and Santos, M.C.F. 2003. Ocorrência de Lysiosquilla glabriuscula (Lamarck, 1818) (Crustacea, Stomatopoda, Lysiosquillidae) no litoral de Pernambuco. Boletim Técnico-Cientifico do CEPENE, 11(1): 293-296.

Dana, J.D. 1852. Crustacea, Part I. United States Exploring Expedition during the years $1838,1839,1840,1841,1842$, under the command of Charles Wilkes, U.S.N., 13: 1-685; Atlas: 1-27, pis. 1-96, 1855, pp. 1-27. C. Sherman, Philadelphia.

Eydoux, A.M. and Souleyet, L. 1842. Zoologie Crustacés. Voyage autour du monde exécuté pendant les années 1836 el 1837 sur la corvette "La Bonite" commandés par $M$. Vaillant, Capitaine de Vaisseau. 1: 219-272.

Fabricius, J.C. 1787. Mantissa insectorum sistens coum species nuper detectas adjectis characieritus nericis, differentiis specificis, emondationibus, observationibus. Christian Gottlieb Proft. Hafniae, 348p.

Fabricius, J.C. 1793. Entomologia systematica, emendata et aucta, Classes, ordines, genera, species adjectis synonimis, locis, observationibus, descriptionibus, 2: i-viii, 1-519.

Fausto Filho, J. 1966. Sôbre a ocorrência de Squilla lijdingi Holthuis, 1959 no litoral Brasileiro (Crustacea, Stomatopoda). Arquivos da Estação de Biologia Marinha da Universidade Federal do Ceará, 6: 139-141.

Fausto Filho, J. 1974. Stomatopod and decapod crustaceans of the Archipelago of Fernando de Noronha, North-east Brazil. Arquivos de Ciências do Mar, 14(1): 1-35.

Fausto Filho, J. 1975. Sobre a ocorrência de Squilla grenadensis Manning, 1969 no litoral Brasileiro (Stomatopoda, Squillidae). Arquivos de Ciências do Mar, 15: 133-135.

Fausto Filho, J. 1978. Crustáceos estomatópodos e decápodos dos substratos de lama do Nordeste brasileiro. Arquivos de Ciências do Mar, 18(1-2): 63-71.

Fausto Filho, J. and Lemos de Castro, A. 1973. Gonodactylus moraisi, nova espécie de crustáceo do Brasil (Stomatopoda: Gonodactylidae). Arquivos de Ciências do Mar, 13(1): 61-63.
Fausto Filho, J. and Sampaio Neto, J.B.S. 1976. Observaçóes sobre alguns crustáceos estomatópodos e decápodos do norte do Brasil. Arquivos de Ciências do Mar, 16: 6571.

Fowler, H.W. 1912. The Crustacea of New Jersey. Report of the New Jerey State Museum, part II, 1911: 29-650.

Gibbes, L.R. 1850. On the carcinological collections of the cabinets of natural history in the United States, with an enumeration of the species contained therein, and descriptions of new species. Proceedings American Association for the Advancement of Science. 3rd meeting: 167-201.

Glassell, S.A. 1934. Some corrections needed in recent carcinological literature. Transactions of the San Diego Society of Natural History. 7: 453-454.

Gomes-Corrêa, M.M. 1986. Stomatopoda do Brasil (Crustacea-Holplocarida). Ph.D. Thesis, Universidade de São Paulo, 320 pp., 35 pls.

Gomes-Corrêa, M.M. 1998. Malacostraca, Hoplocarida. p. 289-398. In: P.S. Young (ed), Catalogue of Crustacea of Brazil. Rio de Janeiro, Museu Nacional. (Série Livros $n^{\circ}$. 6)

Hansen, H.J. 1895. Isopoden, Cumaceen und Stomatopoden der Plankton-Expedition. Ergebnisse der Plankton-Expedition der Humboldt-Stiftung, 2(Gc), 1-105.

Hendrickx, M.E. and Salgado-Barragán J. 2002. Stomatopoda. p. 373-400. In: J.J. Morrone-Lupi; J.E. Llorente-Bousquets and H. Ponce (eds), Biodiversidad taxonomia y biogeografia de artropodos de Mexico: Hacia una sintesis de su conocimiento. Universidad Nacional Autónoma de México, México, D.F, UNAM.

Hernández Aguilera, J.L. and Hermoso Salazar, A.M. 1988. Algunos estomatopodos de la costa este de Mexico con la descripcion de una nueva especie (Crustacea: Stomatopoda). Investigaciones Oceanográficas, serie B 4: $1-13$.

Holmquist, J.G.; Powell, G.V.N. and Sogard, S.M. 1989. Decapod and stomatopod communities of seagrasscovered mud 
banks in Florida Bay: inter- and intrabank heterogeneity with special reference to isolated sub-environments. Bulletin of Marine Science, 44: 251-262.

Holthuis, L.B. 1941. The Stomatopoda of the Snellius Expedition: Biological Results of the Snellius Expedition, XII. Temminckia, 6: 241-294.

Holthuis, L.B. 1959. Stomatopod Crustacea of Suriname. Studies on the Fauna of Suriname and other Guyanas, 10: 173-191.

Holthuis, L.B. 1964. Preliminary note on two new genera of Stomatopoda. Crustaceana, 7: 140-141.

Holthuis, L.B. 1967. The stomatopod Crustacea collected by the 1962 and 1965 Israel South Red Sea Expeditions. The second Israel South Red Sea Expedition, 1965, Report no. 1. Israel Journal of Zoology. 16: 1-45.

Holthuis, L.B. 2000. Nomenclatural notes on eighteenth century Stomatopoda (Hoplocarida). Journal of Crustacean Biology, 20(special number 2): 12-19.

Holthuis, L.B. and Manning, R.B. 1964. Proposed use of the Plenary Powers (A) to designate a type-species for the genera Pseudosquilla Dana, 1852, and Gonodactylus Berthold, 1827, and (B) for the suppression of the generic name Smerdis Leach, 1817 (Crustacea, Stomatopoda). Z.N. (s) 1609. Bulletin of Zoological Nomenclature, 21: 137-143.

Holthuis, L.B.; Edwards, A.J. and Lubbock, H.R. 1980. The decapod and stomatopod Crustacea of St. Paul's Rocks. Zoologische Mededelingen, 56: 27-49.

Ingle, R.W. 1960. Squilla labadiensis n. sp. and Squilla intermedia Bigelow, two stomatopod crusta-ceans new to the West African coast. Annals and Magazine of Natural History, (13)2: 565-576.

Kemp, S. 1913. An account of the Crustacea Stomatopoda of the Indo-Pacific Region based on the collection in the Indian Museum. Memories of Indian Museum, 4: $1-217$.

Kikuchi, R. 2000. The Biological Reserve of "Atol das Rocas". Marine Pollution Bulletin,
41(1/6): 119.

Lamarck, J.B.P.A. 1818. Histoire naturelle des animaux sans vertèbres prsentant les caractéres généraux et particuliers de ces animaux, leur distribution, leur classes, leurs families, leurs genres, et la citation des principales espèces qui s'y rapportent; précédée d'une introduction offrant la détermination des caracteres essentiels, de l'animal, sa distinction du vegetal et des autres corps naturelles, enfin, l'exposition des principes fondamentaux de la zoologie. 5. 1-612. Deterville, Paris.

Leão, Z.M.A.N. and Dominguez, J.M.L. 2000. Tropical coast of Brazil. Marine Pollution Bulletin, 41(1-6): 112-122.

Leach, W.E. 1817-1818. A general notice of the animals taken by Mr. John Cranch, during the expedition to explore the source of the River Zaire. App. 4, pp. 407-419 (1818), 1 pl. (1817). In: J.K. Tuckey (ed.) Narrative of an Expedition to Explore the River Zaire, usually called the Congo in South Africa in 1816, under the direction of Captain J.K. Tuckey. John Murray, London.

Lemos de Castro, A. 1955. Contribuição ao conhecimento dos crustáceos da ordem Stomatopoda do litoral brasileiro (Crustacea, Hoplocarida). Boletim do Museu Nacional do Rio de Janeiro, 128: 1-68.

Linnaeus, C. 1768. Systema naturae per regna tria naturae, secundum classes, ordines, genera, species, cum characteribus, differentiis, synonymis, locis, ed. 12 (3): 1-236. (appendix tomi 1: 23-228), Laurentius Salvius, Stockholm.

Lucatelli, D.; Ahyong, S.T.; Bezerra, L.E.A. and Santos, P.J.P. 2012. A new western Atlantic species of Eurysquilla (Crustacea: Stomatopoda: Eurysquilloidea) with a key to the world species. Journal of the Marine Biological Association of the United Kingdom, 1-5 (on line version).

Lunz, G.R. 1935. The stomatopods (mantis shrimps) of the Carolinas. J. Elisha Mitchell Science and Society. 51: 151-159.

Lunz, G.R. 1937. Stomatopoda of the Bingham Oceanographic Collection. Bulletin of the Bingham Oceanography College, 5(5): 1-19. 
Manning, R.B. 1959. A checklist of the stomatopod crustaceans of the Florida-Gulf of Mexico area. Quarterly Journal of the Florida Academy of Sciences, 22(1): 14-24.

Manning, R.B. 1961. A new Lysiosquilla (Crustacea: Stomatopoda) from the Gulf of California, with a redescription of $L$. decemspinosa Rathbun. Proceedings of the Biological Society of Washington, 74: 29-35.

Manning, R.B. 1962b. Seven new species of stomatopod crustaceans from the northwestern Atlantic. Proceedings of the Biological Society of Washington, 75: 215222.

Manning, R.B. 1963. A new species of Lysiosquilla (Crustacea, Stomatopoda) from the Northern Straits of Florida. Bulletin of Marine Science of the Gulf and Caribbean, 13(1): 54-57.

Manning, R.B. 1963. Preliminary revision of the genera Pseudosquilla and Lysiosquilla with descriptions of six new genera (Crustacea: Stomatopoda). Bulletin of Marine Science of the Gulf and Caribbbean, 13: 308-328.

Manning, R.B. 1966. Stomatopod Crustacea. 3. Campagne de la "Calypso" au Large des côte Atlantiques de l'Amérique du Sud (1961-1962). Annales de l'Institut océanographique Mônaco, 44: 359-384.

Manning, R.B. 1967. Review of the Genus Odontodactylus (Crustacea: Stomatopoda). Proceedings of the United States National Museum, 123(3606): 1-35.

Manning, R.B. 1968. Stomatopod Crustacea from Madagascar. Proceedings of the United States National Museum, 124 (3641): 1-61.

Manning, R.B. 1969. Stomatopod Crustacea of the Western Atlantic. Studies in Tropical Oceanography, 8: viii-380.

Manning, R.B. 1970. Nine new American Stomatopod Crustaceans. Proceedings of the Biological Society of Washington, 83: 99-114.

Manning, R.B. 1974. Stomatopods collected by Th. Mortensen in the Eastern Pacific region (Crustacea, Stomatopoda). Steenstrupia, 3: 101-109.

Manning, R.B. 1977. A Monograph of the
West African Stomatopod Crustacea. Atlantide Report, 12: 25-181.

Manning, R.B. 1980. The superfamilies, families, and genera of recent stomatopod Crustacea, with diagnoses of six new families. Proceedings of the Biological Society of Washington, 93: 362-372.

Manning, R.B. 1995. Stomatopod Crustacea of Vietnam: The Legacy of Raoul Serine. Crustacean Research, special number 4: vii339.

Manning, R.B. and Bruce, A.J. 1984. Erythrosquilla megalops, a remarkable new stomatopod from western Indian Ocean. Journal of Crustacean Biology, 4: 324-332.

Manning, R.B. and Camp, D.K. 1993. Erythrosquilloidea, a new superfamily, and Tetrasquillidae, a new family of stomatopod crustaceans. Proceedings of the Biological Society of Washington, 106: 85-91.

Manning, R.B. and Chace, F.A. 1990. Decapod and stomatopod Crustacea from Ascension Island, South Atlantic Ocean. Smithsonian Contributions to Zoology, 503: 1-91.

Manning, R.B. and Heard, R.W. 1997. Stomatopod crustaceans from the Carolinas and Georgia, Southeastern United States. Gulf Research Report, 9(4): 303-320.

Manning, R.B. and Lewinsohn, C. 1986. Notes on some stomatopod crustacea from the Sinai Peninsula, Red Sea. Smithsonian Contributions to Zoology, 433: 1-19.

Miers, E.J. 1880. On the Squillidae. Annals and Magazine of Natural History, (5)5: 1-30, 108-127, pls. 1-3.

Milne-Edwards, A. 1878. Description de quelques espèces nouvelles de crustacés provenant du voyage aux îles du Cap Vert de MM Bouvier et de Cessac. Bulletin de la Société Philomathique de Paris, 2: 225-232.

Milne Edwards, H. 1837. Histoire naturelle de crustacés, comprenant l'anatomie, la physiologie et la classification de ces animaux, 2: 1-532.

Moosa, M.K. 1991. The Stomatopoda of New Caledonia and Chesterfield Islands. p. 147219. In: Le benthos des fonds meubles des lagons de Nouvelle-Calédonie, Vol. 1 (B. Richer de Forges, ed.), Ed. Orstom. 
Moosa, M.K. 2000. Marine biodiversity of the South China Sea. Raffles Bulletin of Zoology, 8(Supplement): 405-457.

Morgan, S.G. and Goy, J.W. 1987. Reproduction and larval development of the mantis shrimp Gonodactylus bredini (Crustacea: Stomatopoda) maintained in the laboratory. Journal of Crustacean Biology, 7(4): 595-618.

Ortiz, M. and Silva S. 1992. Lista de especies y bibliografia de los crustaceos de la Republica popular de Mozambique. Revista de Investigaciones Marinas, 13: 9-38.

Rathbun, M.J. 1935. Fossil Crustacea of the Atlantic and Gulf coastal plain. Geological Society of America, Special 2: 1-160.

Reaka, M.L. and Manning, R.B. 1980. The distributional ecology and zoogeographical relationships of stomatopod Crustacea from Pacific Costa Rica. Smithsonian Contributions to the Marine Sciences, 7: 1-29.

Reaka, M.L. and Manning, R.B. 1987. Stomatopod Crustacea of Enewetak Atoll. p 181-190. In: D.M. Devaney; E.S. Reese; B.L. Burch and P. Helfrich (eds). The Natural History of Enewetak Atoll, Vol. II. Biogeography and Systematics. Office of Scientific and Technical Information U.S. Dept. Energy, Washington, D.C.

Richer de Forges, B. and Moosa, M.K. 1992. Distribution of stomatopods (Crustacea) in the lagoons of New Caledonia and Chesterfield Atoll. Raffles Bulletin of Zoology, 40: 149-162.

Rodrigues, S.A. 1966. Ocorrência de Acanthosquilla floridensis Manning na Costa de São Paulo. Ciência e Cultura, 18: 142.

Rodrigues, C. and Young, P.S. 2005. Stomatopoda (Crustacea, Hoplocarida) coletados pelo Programa REVIZEE com duas novas ocorrências para a costa do Brasil. Arquivos do Museu Nacional, 63(2): 233-245.

Say, T. 1818. An account of the Crustacea of the United States. Journal of the Academy of Natural Sciences of Philadelphia, 1: 235253.

Schmitt, W.L. 1924. Report on the Macrura,
Anomura and Stomatopoda collected by the Barbados-Antigua Expedition from the University of Iowa in 1918. Studies in Natural History University, 10(4): 65-99.

Schmitt, W.L. 1940. The stomatopods of the west coast of America based on collections made by the Allan Hancock Expeditions, 1933-1938. Allan Hancock Foundation Pacific Expedition, 5: 129-225.

Schotte, M. and Manning, R.B. 1993. Stomatopod Crustacea from Tobago, West Indies. Proceedings of the Biological Society of Washington, 106: 566-581.

Schram, F.R. 2010. Catalog of the fossil and recent Stomatopoda. Langley, Washington, Bay Ridge Press, 294p.

Silva, K.C.A; Muniz, A.P.M.; Viana, G.F.S.; Cintra, I.H.A. and Ramos-Porto, M. 2003. Espécies de estomatópodes capturadas na pesca industrial do camarão-rosa e no programa REVIZEE, na região Norte do Brasil (Crustacea, Stomatopoda). Boletim Técnico-Cientifico do CEPNOR, 3(1): 3751.

Stimpson, W. 1857. Stomatopoda. In: On the Crustacea and Echinodermata of the Pacific shores of North America. Boston Journal of Natural History, 6(27): 444-532.

Viana, G.F.S; Silva, K.C.A; Cintra, I.H.A. and Ramos-Porto, M. 1998. Novos registros de Stomatopoda (Crustacea: Hoplocarida) para a costa Norte Brasileira coletados durante o Programa REVIZEE. Trabalhos Oceanográficos da Universidade Federal de Pernambuco, 26(1): 99-102.
Submitted 07 May 2012 Accepted 31 October 2012 